\title{
Ultrasonic treatment of polycarbonate/carbon nanotubes composites
}

\author{
Xiang Gao, Avraam I. Isayev*, Chao Yi \\ Department of Polymer Engineering, The University of Akron, Akron, OH 44325-0301, USA
}

*Corresponding author. Tel.: +1 (330)972-6673.

E-mail address: aisayev@uakron.edu (A.I. Isayev).

\begin{abstract}
A twin-screw extruder having an ultrasonic treatment zone in the barrel was used to prepare polycarbonate (PC)/multi-walled carbon nanotubes (MWNTs) composites. The effect of molecular weight of PC, carbon nanotubes (CNTs) concentration and ultrasonic amplitude on processing characteristics, electrical, morphological, rheological and mechanical properties of PC filled with 0.2-1.5 wt\% CNTs was investigated. Ultrasonic treatment showed significant effects on improving the dispersion and distribution of CNTs especially in low molecular weight PC (LPC). The electrical and rheological percolation thresholds of low and high molecular weight PCs obtained by fitting the experimental data to classic percolation theory were significantly decreased after ultrasonic treatment. Specifically, the electrical percolation threshold of LPC/CNTs composites decreased from 0.176 vol\% (0.30 wt $\%$ ) for untreated samples to $0.088 \mathrm{vol} \%(0.15 \mathrm{wt} \%)$ for treated samples at an amplitude of $13 \mu \mathrm{m}$. To the best of our knowledge, this obtained value is the lowest percolation threshold, so far, achieved in melt processed PC/CNTs composites. The mechanism for the decrease of rheological and electrical percolation thresholds after ultrasonic treatment was proposed. Also, some improvements in mechanical properties (including the Young's modulus and elongation at break) of PC/CNTs composites after ultrasonic treatment at an amplitude of $13 \mu \mathrm{m}$ were observed. The ultrasonic extrusion technology is shown to be a suitable method for manufacturing high quality products at lower CNTs concentration.
\end{abstract}

Keywords: polycarbonate, molecular weight, carbon nanotubes, ultrasound, percolation 


\section{Introduction}

Since the discovery of CNTs in 1991 by Iijima [1], CNTs have drawn much attention due to their high aspect ratio, extraordinary mechanical, electrical and thermal properties [2-6]. It has been theoretically and experimentally proved that individual single-wall carbon nanotubes (SWNTs) showed tensile modulus of $640 \mathrm{GPa}$ to $1 \mathrm{TPa}$ [7]. The tensile strength of multi-wall carbon nanotubes (MWNTs) was found to be $\sim 63 \mathrm{GPa}$ [8]. The electrical conductivity of CNTs can be as high as $9.2 \times 10^{4} \mathrm{~S} / \mathrm{m}$ [9]. Also, the thermal conductivity of CNTs can reach up to $6600 \mathrm{~W} / \mathrm{m} \cdot \mathrm{K}$ at a room temperature [10]. All these extraordinary properties make CNTs the best filler candidates to prepare high performance polymer composites, especially conductive polymer composites. Though the price of CNTs is steadily decreasing over years, it is still significantly more expensive than traditional carbon fillers, like carbon black. Thus to lower the cost of products, it would be beneficial to reduce the electrical percolation threshold of conductive polymer composites. The percolation threshold is the critical concentration of a conductive filler at which a conductive network is formed, exhibiting a sudden jump of the electrical conductivity [11]. After the percolation threshold, the electrical conductivity will not change much. Therefore, reaching the electrical percolation threshold at low filler concentration could provide the polymer/CNTs composites with electrical conductivity being sufficient to meet most of the applications, such as electromagnetic interface (EMI) shielding and antistatic materials. Also, decreasing the percolation threshold can help to improve the processability of the composites and maintain or even improve the mechanical properties of the composites, since high loading of CNTs may induce more stress concentrators, diminishing the elongation and tensile stress of the composites. In order to decrease the electrical percolation, better dispersion of nanotubes in the polymer composites is required. However, due to the very large van der Waals force between the nanotubes and large number of primary agglomerates existing in the raw CNTs, it is very difficult to disperse CNTs in polymer composites, which makes the efficient transition of nanotube properties to the polymer matrix challenging [12].

The commonly used methods to prepare polymer composites are in-situ polymerization [13-16], solution methods [17-19], and melt processing [3-5,20-22]. Though good dispersion of CNTs can be achieved by in-situ polymerization, there are only limited numbers of polymers that can be used in in-situ polymerization. Solution methods are highly dependent on heavy use of chemicals and it is not easy to scale up. Compared with these two methods, melt compounding is a promising method to disperse nanofillers in polymer composites. It is efficient and environmentally friendly. Also, from a product commercialization perspective, it is most preferred because it can be 
easily scaled up. However, one drawback of melt processing method is that the dispersion of nanofillers in the products is not as good as using the other two methods. Over the past decade, high power ultrasound has been proven to be an efficient method to improve the dispersion of nanofillers in polymer matrix [4,20-25]. Swain and Isayev studied the high density polyethylene (HDPE)/clay nanocomposites prepared by a single screw compounding extruder attached with an ultrasound die. It was found that the intercalation of HDPE into lattice layers was enhanced and the oxygen permeability of the nanocomposites was decreased after ultrasonic treatment [22]. Zhong et al. studied the polypropylene (PP)/CNTs composites using ultrasonically aided twin-screw extruder. After ultrasonic treatment, better dispersion of CNTs was achieved and electrical, mechanical properties were improved [4].

Polycarbonate (PC) is a widely used engineering plastic exhibiting high transparency, high impact-resistance and excellent flexibility over a wide temperature range. Preparation of PC/CNTs composites using melt processing has been extensively studied [26-28]. The effect of screw configuration of twin-screw extruder [26], polymer viscosity [27], and types of CNTs [28] were studied. It was found that the aspect ratio of CNTs was critical to achieve lower percolation [29]. Longer residence time and higher polymer viscosity in extrusion could break up more CNTs agglomerates, but the nanotubes might be also broken up at the same time, resulting in reduction of their aspect ratio. Most of the electrical percolation thresholds of $\mathrm{PC} / \mathrm{CNT}$ composites in these works were reported to lay between $0.3 \mathrm{wt} \%$ and $0.5 \mathrm{wt} \%$ [27,28]. As for the mechanical properties of PC/CNTs composites, little improvement was achieved with the addition of CNTs in PC composites [28,30].

In the present study, ultrasonically aided twin-screw extruder was used to prepare PC/CNTs composites. Two different molecular weight PCs were used to study the effect of molecular weight on the electrical, morphological and mechanical properties of ultrasonically treated polymer composites. Rheological properties of the PC/CNTs composites were also investigated to explain the effect of ultrasonic treatment on the properties of composites. The electrical and rheological percolation thresholds were obtained by fitting the experimental data to the classic percolation theory. High power ultrasonic device placed in the barrel was found to be an easy and efficient method to improve the dispersion and distribution of CNTs and decrease the electrical percolation threshold of the PC/CNTs composites to the level not seen in the available literature. The mechanical properties of the PC/CNTs composites were also studied. Finally, the mechanism of the decrease of percolation threshold was proposed. 


\section{Experimental}

\subsection{Materials}

Polycarbonate Lexan OQ3820 with high molecular weight $\left(\mathrm{M}_{\mathrm{w}}=31,110 \mathrm{~g} / \mathrm{mol}\right.$ by GPC, denoted as HPC) and polycarbonate Lexan OQ1030 with low molecular weight $\left(\mathrm{M}_{\mathrm{w}}=17,610 \mathrm{~g} / \mathrm{mol}\right.$ by GPC, denoted as LPC) were kindly provided by SABIC plastics. These two PCs have linear structure. The multi-walled carbon nanotubes (CNTs) produced by catalytic carbon vapor deposition (CCVD) process were provided by Nanocyl S.A. (Belgium), under the trade name of NC7000. According to the producer, it has carbon purity of $>90$ wt $\%$, average diameter of 9.5 $\mathrm{nm}$, average length of $1.5 \mu \mathrm{m}$, surface area of $250-300 \mathrm{~m}^{2} / \mathrm{g}$ by BET method, bulk density of $51 \mathrm{~g} / \mathrm{L}$, density of 2.04 $\mathrm{g} / \mathrm{cm}^{3}$ by Helium Pycnometry. CNTs are used as received in this study.

\subsection{Sample preparation}

PC easily absorbs moisture, which may influence the properties of PC after thermal processing. The allowable moisture content for molding a satisfactory part is $0.03 \%$ [31]. Therefore, prior to all thermal processing (extrusion, compression molding, injection molding), $\mathrm{PC}$ pellets were dried in a vacuum oven at $100^{\circ} \mathrm{C}$ overnight to ensure low moisture content.

A co-rotating twin-screw extruder (PRISM USALAB 16, Thermo Electron Corp. UK) with an ultrasonic treatment zone located in the extruder barrel, as seen in Fig. 1(a), was used for composites preparation. The screw configuration used in this study is shown in Fig. 1(b). The screw consists of conveying sections, kneading sections and an ultrasonic zone. The extruder has a screw diameter of $16 \mathrm{~mm}$ and an L/D of 25. Compounding was carried out at a screw speed of $200 \mathrm{RPM}$, a feeding rate of $908 \mathrm{~g} / \mathrm{hr}(2 \mathrm{lbs} / \mathrm{hr})$, and a temperature profile of 240, 260, 285, $285,285,285^{\circ} \mathrm{C}$ for $\mathrm{HPC}$ composites, $210,225,240,240,240,240^{\circ} \mathrm{C}$ for $\mathrm{LPC}$ composites from the hopper to the die. PC pellets were fed into the extruder by feeder 1 and CNTs were fed downstream by feeder 2 located at position where PC pellets were already melted. In this way, the breakage of CNTs caused by solid PC particles can be reduced. After feeding of CNTs, the composites material continued downstream through a mixing section (KN2 in Fig. 1(b)) before it entered the ultrasonic treatment zone. After ultrasonic treatment, the composites were further mixed (KN3 in Fig. 1(b)) and then exited through a circular die. The strand was subsequently water quenched and pelletized. The ultrasonic system (Branson Ultrasonic Corp., CT, USA) works at a frequency of $40 \mathrm{kHz}$. Power 
consumption was measured using a Dataq data acquisition system connected directly to the power supply. The torque of the extruder was recorded as the percentage of the maximum extruder torque being $100 \%$. Untreated and treated samples at ultrasonic amplitudes of $7.5,10,13 \mu \mathrm{m}$ with CNTs concentrations of $0,0.2,0.3,0.4,0.8,1.5 \mathrm{wt} \%$ were prepared. The samples at different concentrations were denoted as PC-xCNT with $\mathrm{x}$ being the weight concentration of CNTs.

(a)

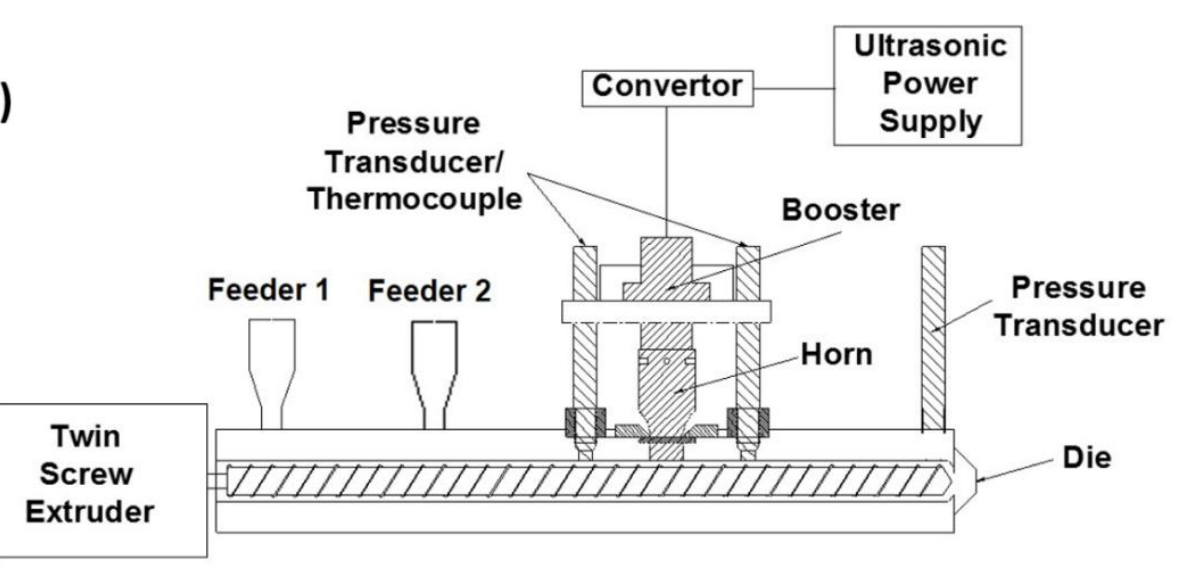

(b)

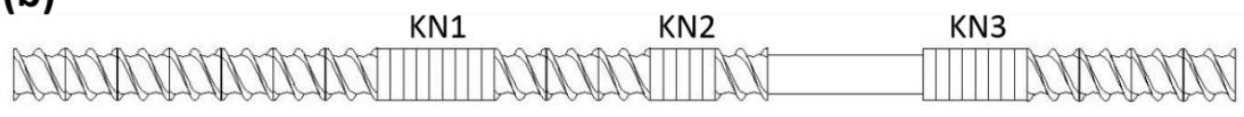

Fig. 1. Schematic of the ultrasonic twin-screw extruder (a) and the screw configuration (b) used in this study.

\subsection{Electrical resistivity}

Samples for electrical resistivity test with a diameter of $90 \mathrm{~mm}$ and a thickness of $1 \mathrm{~mm}$ were prepared by compression molding at $250^{\circ} \mathrm{C}$ and $49 \mathrm{MPa}$ using a compression molding machine (CARVER 4122, Wabash, IN, USA). A Keithley electrometer (Model 6517A, Keithley Instruments, Cleveland, OH, USA) with an 8009 test fixtures was used to measure the volume resistivity of the composites in the range from $10^{18}$ to $10^{7} \mathrm{Ohm} \cdot \mathrm{cm}$ in accordance with the ASTM D257. Alternating polarity mode using a voltage of \pm 10 volts was used to eliminate the influence of background current. For samples with volume resistivity lower than $10^{7} \mathrm{Ohm} \cdot \mathrm{cm}$, a Keithley Microohmmeter (Model 580, Keithley Instruments, Cleveland, OH, USA) was used for both the input and output signals in accordance with the ASTM 4496.

The Keithley electrometer 6517A with 8009 test fixtures was also used to measure the surface resistivity of the composites in the range from $10^{15}$ to $10^{7} \mathrm{Ohm} /$ square. Van der Pauw four-point probe method was used to 
characterize the surface resistivity of the samples lower than $10^{7} \mathrm{Ohm} / \mathrm{square}$. Two Keithley 2400 source meters were used during the test and four tungsten probes connected to the source meters were placed on top of the four silver coated corners of the sample [5]. Two probes were used to apply the current and the other two probes were used to measure the voltage. The data was collected by computer through the Labview softwares.

\subsection{Rheological measurements}

An ARES G2 strain-controlled rotational rheometer (TA Instruments) with parallel plate geometry was used to study the rheological properties of the composites. Circular disk samples with a diameter of $25 \mathrm{~mm}$ and a thickness of $2 \mathrm{~mm}$ were prepared by compression molding at $250{ }^{\circ} \mathrm{C}$ and $49 \mathrm{MPa}$. These samples were then loaded to the parallel plates in the rheometer and squeezed to $1.8 \mathrm{~mm}$. All the tests were conducted at strain amplitudes in the linear viscoelastic region at a constant temperature of $230{ }^{\circ} \mathrm{C}$ for HPC and $210{ }^{\circ} \mathrm{C}$ for LPC composites. Different testing temperatures were used for HPC and LPC in order to observe the terminal behavior of the composites which was highly influenced by the dispersion state of CNTs. The storage modulus, loss modulus and loss tangent were recorded in a frequency range from 0.05 to $200 \mathrm{rad} / \mathrm{s}$.

\subsection{Morphological study}

The dispersion of CNTs in the scale of several micrometers was studied using an optical microscope (Olympus BX51 Microscope System). The sample was prepared by following procedures. Firstly, a very small piece of composites was cut from a randomly chosen extruded pellets and put between two cover glasses. Then, the glasses were put onto the lower hot face $\left(250^{\circ} \mathrm{C}\right)$ of compression molding machine and the upper cover glass was pressed slowly with a tweezer, making the sample being hot pressed into a very thin film with a thickness of about $10 \mu \mathrm{m}$. The sample was then moved to optical microscopy for observation. Transmission electron microscope (TEM) (JEOL JEM-1230, Tokyo, Japan) was used to investigate the nanoscale dispersion of CNTs in the composites. TEM samples with a thickness of about $100 \mathrm{~nm}$ were cut from an injection molded dumbbell sample with a Leica EM UC7 microtome machine. It needs to be mentioned that the TEM pictures were randomly taken in more than three specimens and therefore pictures shown here represent the dispersion state of CNTs in the samples.

\subsection{Tensile testing}


The tensile test samples were injection molded with a Haake MiniJet Injection Molding System (Thermo Scientific, USA). Due to the difference in melt viscosity of HPC and LPC composites, different cylinder and mold temperatures were used. A cylinder temperature of $330{ }^{\circ} \mathrm{C}$ and a mold temperature of $130{ }^{\circ} \mathrm{C}$ were used for HPC composites, and a cylinder temperature of $300{ }^{\circ} \mathrm{C}$ and a mold temperature of $110^{\circ} \mathrm{C}$ were used for LPC composites. An injection pressure and packing pressure of $50 \mathrm{MPa}$ was used for both HPC and LPC composites. Tensile tests were conducted on an Instron Testing Machine (Model 5567, Instron Corp.) with $1 \mathrm{kN}$ load cell at room temperature according to ASTM 638 (type V) without extensometer. A crosshead speed of $50 \mathrm{~mm} / \mathrm{min}$ was used and the reported results were averages of at least six samples. The Young's modulus, yield strength, elongation at break and tensile strength were measured.

\section{Results and Discussion}

\subsection{Processing characteristics}

The power consumption of HPC and LPC composites during ultrasonic treatment as a function of CNTs concentration is plotted in Fig. 2. The measured power consumption is the total power consumption during the treatment of composites. Part of it was dissipated as heat and the rest was exerted to break the agglomerate of CNTs, improving the dispersion of CNTs in polymer melt. As seen from Fig. 2, the power consumption increased with ultrasonic amplitude, because the increase of amplitude caused an increase of the acoustic pressure, resulting in the increase of power consumption [32]. Higher power consumption indicated that more energy was transmitted to polymer melt. Especially, there was a large increase of power consumption from $7.5 \mu \mathrm{m}$ to $10 \mu \mathrm{m}$.

Little difference of power consumption between samples at different CNTs concentration was observed. The average value of power consumption of LPC at same CNTs concentration and ultrasonic amplitude was higher than that of HPC composites. A theoretical modeling of ultrasonic power consumption in a bubble filled polymer indicated that the power consumption increased with sound velocity, hydrostatic pressure and longitudinal loss tangent of material at ultrasonic frequency [33]. The relatively higher power consumption in LPC composites was possibly due to the relatively higher value of loss tangent at the ultrasound frequency and difference of hydrostatic pressure at the ultrasonic treatment zone. 

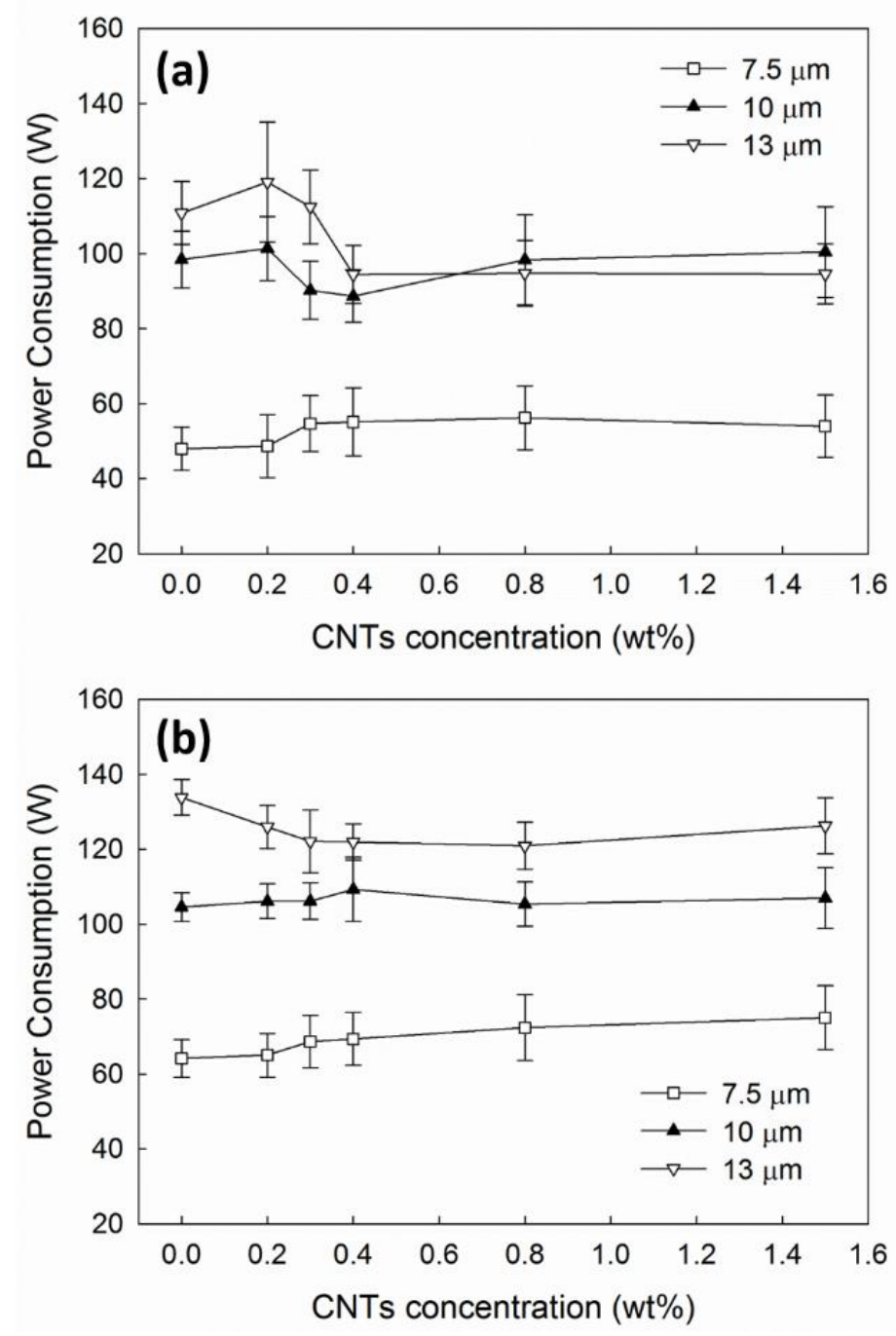

Fig. 2. Ultrasonic power consumption of (a) HPC and (b) LPC composites.

A continuous decrease of torque was observed with increasing ultrasonic amplitude, as shown in Fig. 3. This torque decrease could be caused by permanent effect including the degradation of polymer under action of ultrasound and the thixotropic effects including the shear thinning in the melt. The decrease of torque is beneficial in polymer melt processing by improving the processability of high viscosity polymers. Taking the error bar of the torque data into consideration, there was little change of the torque at different CNTs concentrations. This was due to the thixotropic effect caused by ultrasonic treatment and the high shear rate in the extruder. When comparing the torque of HPC and LPC composites, the torque of HPC at same CNTs concentration and ultrasonic amplitude was higher than that of LPC composites. This was caused by the higher viscosity of HPC composites during extrusion. 

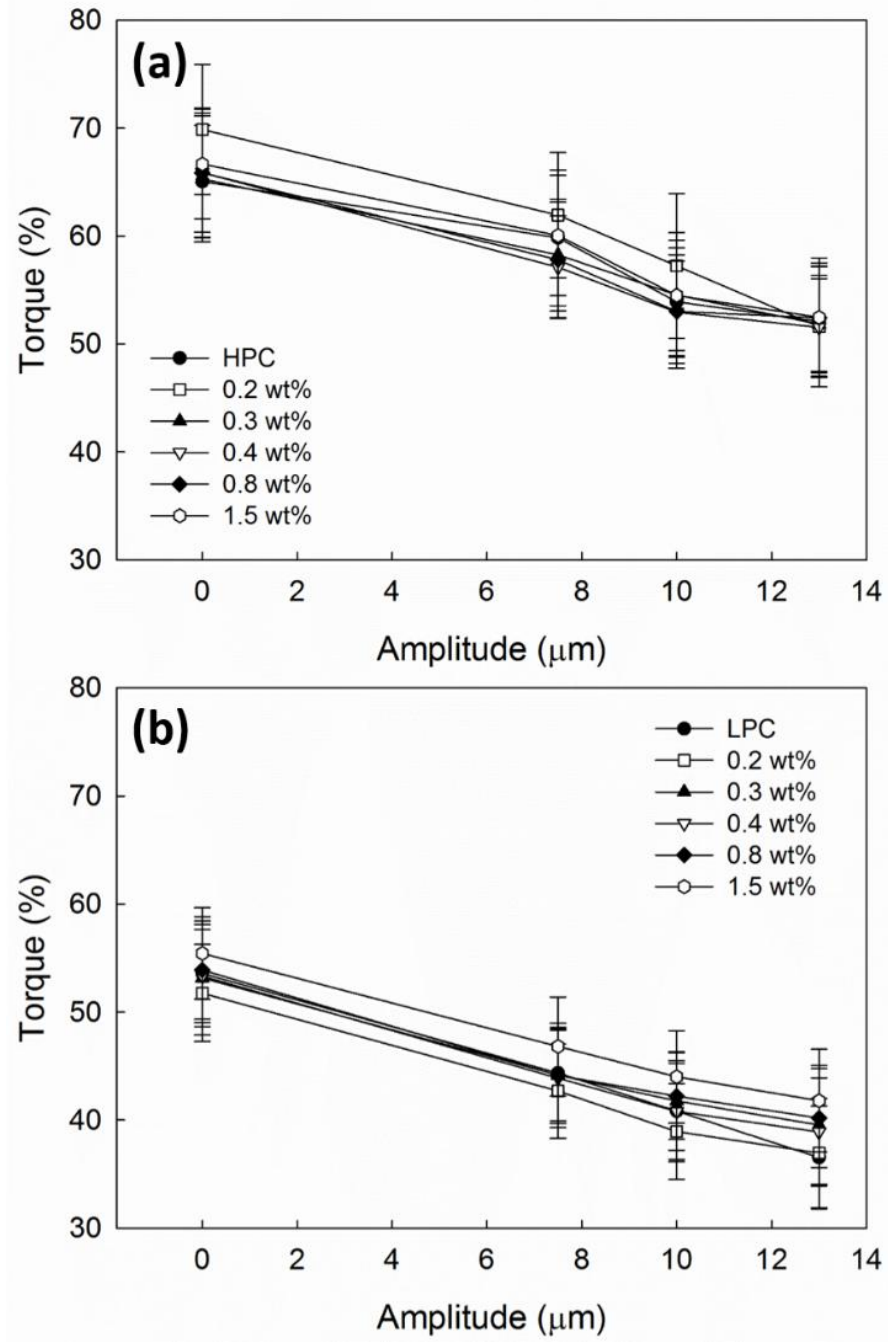

Fig. 3. Torque of the twin-screw extruder of HPC (a) and LPC (b) composites.

\subsection{Electrical properties}

The surface resistivity of the PC/CNTs composites were measured and plotted in Fig. 4. In general, the surface resistivity of the composites continuously decreased with the increase of CNTs concentration. A sudden decrease of the resistivity can be observed while percolation threshold was reached. Ultrasonic treatment showed a larger effect on decreasing the surface resistivity for low CNTs concentration samples. The electrical percolation threshold continuously decreases with the increase of ultrasonic amplitude. 

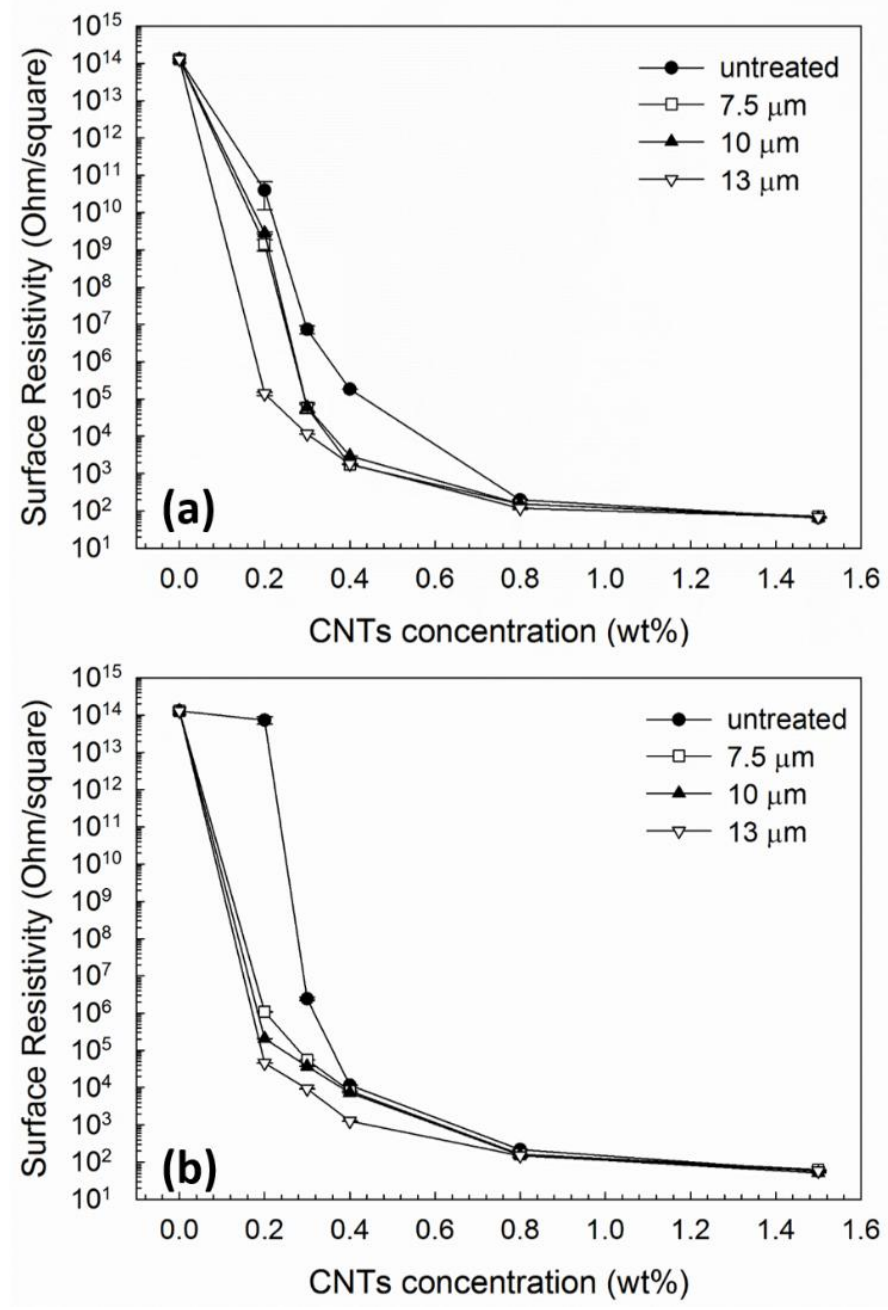

Fig. 4. Surface resistivities of HPC (a) and LPC (b) composites.

The volume resistivities of the composites are plotted in Fig. 5, showing similar trend as the surface resistivity. Specifically, the volume resistivity continuously decreased with the increase of CNTs concentration and the electrical percolation thresholds decreased with the increase of ultrasonic amplitude. Furthermore, it can be seen from Fig 5(a) that the electrical percolation threshold for HPC composites treated at an amplitude of $13 \mu \mathrm{m}$ was significantly decreased compared with the untreated sample and samples treated at $7.5 \mu \mathrm{m}$ and $10 \mu \mathrm{m}$. Also, as seen from Fig. 5(b), LPC composites showed an even more obvious effect of ultrasound on the electrical percolation threshold. At $0.2 \mathrm{wt} \%$, the samples treated at $7.5 \mu \mathrm{m}$ were already percolated and showed a large decrease of the volume resistivity compared to the untreated samples. 

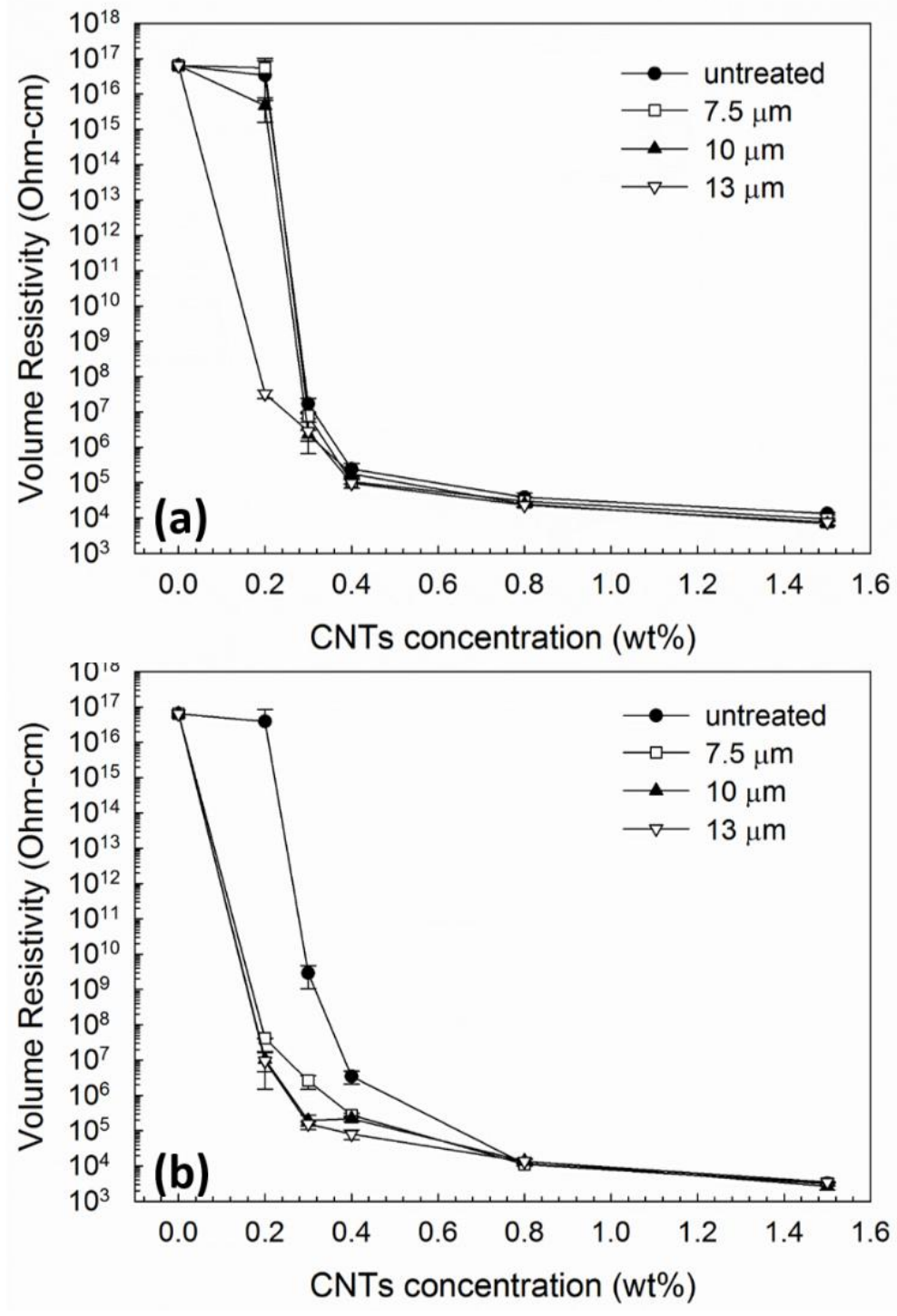

Fig. 5. Volume resistivities of HPC (a) and LPC (b) composites.

In order to fit the volume resistivity data using classic percolation theory, the volume resistivity $\rho(\mathrm{Ohm} \cdot \mathrm{cm})$ was converted to electrical conductivity $\sigma(\mathrm{S} / \mathrm{cm})$ with following equation:

$$
\sigma=1 / \rho
$$

The following equation was used to fit the electrical conductivity, $\sigma$, of the composites in order to obtain the percolation threshold of the composite at various ultrasonic amplitudes [34]:

$$
\sigma(\varphi)=k\left(\varphi-\varphi_{0}\right)^{v}
$$


where $\varphi$ is the volume concentration of CNTs, $\varphi_{0}$ is the electrical percolation threshold, $v$ is the exponent. According to the classic percolation theory, when the fillers forms a three dimensional network in the matrix, i.e. the percolated network in the PC/CNTs composites, the value of $v$ equals $2[11,35]$. Therefore, $v=2$ was used in the further fitting.

In order to fit the data to Eq. (2), the weight fractions were converted to volume fractions as shown in Table 1, based on the density of PC of $1.2 \mathrm{~g} / \mathrm{cm}^{3}$ and the density of CNTs of $2.04 \mathrm{~g} / \mathrm{cm}^{3}$ provided by manufacturer.

Table 1 The weight and volume fractions of CNT in PC/CNT composites

\begin{tabular}{cccccc}
\hline wt\% & 0.2 & 0.3 & 0.4 & 0.8 & 1.5 \\
\hline vol\% & 0.118 & 0.177 & 0.236 & 0.472 & 0.888 \\
\hline
\end{tabular}

Composites with CNTs concentration above the percolation threshold were chosen to fit the electrical conductivity to Eq. (2). The results are shown in Fig. 6 with the inset figure sample showing fitting of the conductivity data for samples treated at $13 \mu \mathrm{m}$. The fitting parameters are shown in Table 2. The electrical percolation thresholds in Table 2 are plotted in Fig. 7.

According to Table 2, the electrical percolation threshold of HPC and LPC composites continuously decreased with the increase of ultrasonic amplitude. The $\varphi_{0}$ of HPC composites decreased from 0.135 vol\% $(0.23$ wt $\%)$ for untreated samples to $0.109 \mathrm{vol} \%(0.19 \mathrm{wt} \%)$ for treated samples at an amplitude of $13 \mu \mathrm{m}$. The $\varphi_{0}$ of LPC composites decreased from 0.176 vol\% $(0.30 \mathrm{wt} \%)$ for untreated samples to 0.088 vol\% $(0.15$ wt $\%)$ for treated samples at an amplitude of $13 \mu \mathrm{m}$. When comparing data of HPC and LPC composites, it can be observed that the electrical percolation threshold of untreated HPC composite was lower than that of untreated LPC composite. The shear stress of HPC composite during extrusion was higher than that of LPC composite (as indicated by higher torque shown in Fig. 3), resulting in better CNTs dispersion (as indicated by the morphological results that will be discussed later). However, the $\varphi_{0}$ of LPC composites decreased faster than HPC composites and resulted in a lower percolation threshold for ultrasonically treated samples. This indicated that ultrasound showed larger effect on improving the electrical properties of LPC composite. To the best of the authors' knowledge, the electrical percolation threshold of $0.088 \mathrm{vol} \%(0.15 \mathrm{wt} \%)$ for treated LPC/CNTs composites at $13 \mu \mathrm{m}$ is the lowest percolation threshold obtained in melt processed PC/MWNTs composites $[11,28,30]$. 

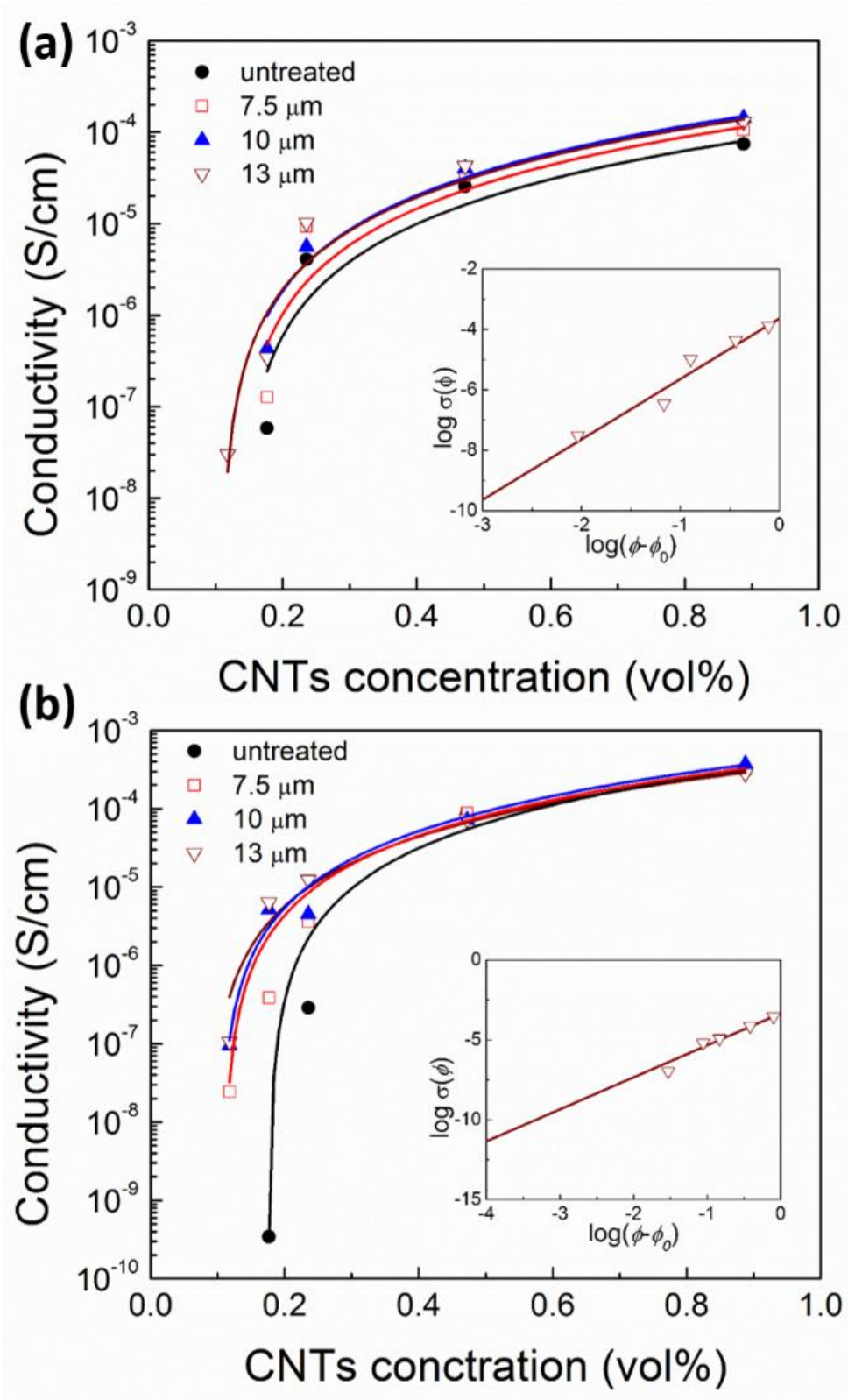

Fig. 6. Electrical conductivity of HPC (a) and LPC (b) composites as a function of volume concentration of CNTs at different ultrasonic amplitudes. Inset figure shows the fitting of PC/CNTs composites treated at an amplitude of 13 $\mu \mathrm{m}$. 
Table 2. Fitting parameters of Eq. (2) for HPC and LPC composites.

\begin{tabular}{c|c|cccc}
\hline \multicolumn{2}{c}{} & $0 \mu \mathrm{m}$ & $7.5 \mu \mathrm{m}$ & $10 \mu \mathrm{m}$ & $13 \mu \mathrm{m}$ \\
\hline \multirow{3}{*}{$\mathrm{HPC}$} & $\mathrm{k}(\mathrm{S} / \mathrm{m})$ & $1.42 \mathrm{E}-04$ & $1.96 \mathrm{E}-04$ & $2.50 \mathrm{E}-04$ & $2.28 \mathrm{E}-04$ \\
& $\varphi_{0}(\mathrm{vol} \%)$ & 0.135 & 0.127 & 0.114 & 0.109 \\
& $\mathrm{R}^{2}$ & 0.9090 & 0.9020 & 0.9731 & 0.9273 \\
\hline \multirow{3}{*}{$\mathrm{LPC}$} & $\mathrm{k}(\mathrm{S} / \mathrm{m})$ & $6.23 \mathrm{E}-04$ & $5.38 \mathrm{E}-04$ & $5.90 \mathrm{E}-04$ & $4.54 \mathrm{E}-04$ \\
& $\varphi_{0}(\mathrm{vol} \%)$ & 0.176 & 0.110 & 0.104 & 0.088 \\
& $\mathrm{R}^{2}$ & 0.9086 & 0.9311 & 0.9732 & 0.9876 \\
\hline
\end{tabular}

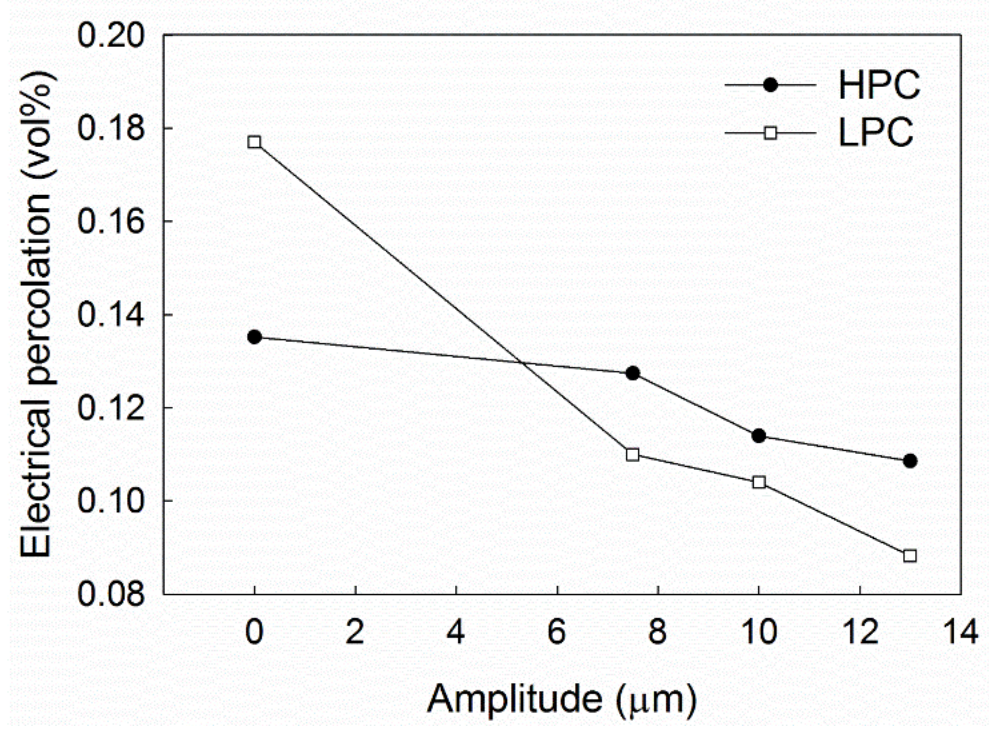

Fig. 7. Electrical percolation threshold as a function of ultrasonic amplitude of HPC and LPC composites.

\subsection{Morphology}

The decrease of the electrical percolation after ultrasonic treatment can be attributed to the improved CNTs dispersion and distribution in the composites. Optical microscopy was used to characterize the dispersion of CNTs in the scale of micrometers. The optical micrographs of HPC-1.5CNT and LPC-1.5CNT samples untreated and treated at an amplitude of $13 \mu \mathrm{m}$ are shown in Fig. 8, indicating agglomerates present in composites. Little and significant differences between untreated and treated samples were observed in HPC-1.5CNT and LPC-1.5CNT, respectively. A significant improvement of CNTs distribution in LPC-1.5CNT after ultrasonic treatment could be 
observed by comparing Fig. 8(c) and (d). Smaller sizes and less number of CNTs agglomerates were observed in treated LPC-1.5CNT sample. Even for the samples with highest CNTs concentration in this study (1.5 wt\%), very few CNTs agglomerates were found in the optical micrographs, showing that CNTs can be dispersed very well in PC. This was also observed in the study by Socher et al. [27]. Less difference was seen in the HPC and LPC composites at lower CNTs concentrations from optical microscopy (not shown here) due to its low magnification. In order to see changes in the dispersion at higher magnification, TEM was used. In fact, TEM was proven to be an efficient method to characterize the dispersion of CNTs in the nanometer scale [36,37]. Accordingly, TEM was used to characterize the dispersion of CNTs for PC- $0.2 \mathrm{CNT}$ and PC- $0.8 \mathrm{CNT}$ samples untreated and treated at an amplitude of $13 \mu \mathrm{m}$, as shown in Figs. 9 and 10. It can be seen that in HPC-0.2CNT, before ultrasonic treatment, there were some agglomerates in Fig. 9(a). After ultrasonic treatment at an amplitude of $13 \mu \mathrm{m}$, the agglomerates of CNTs were broken up and very few agglomerates can be seen in Fig. 9(b). This explains why the decrease of

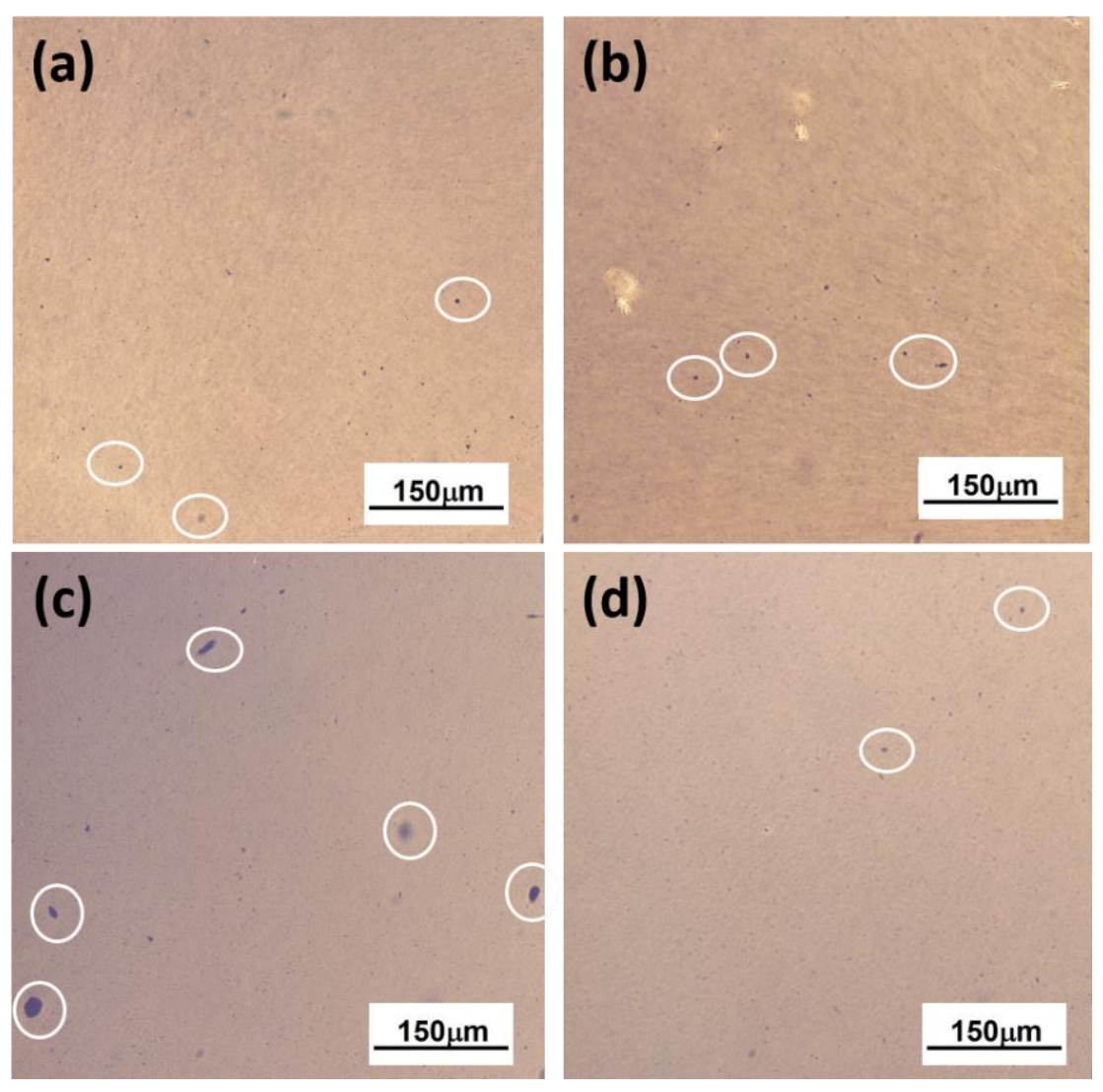

Fig. 8. The optical micrographs of HPC-1.5CNT sample untreated (a) and treated at an amplitude of $13 \mu \mathrm{m}$ (b) and LPC-1.5CNT sample untreated (c) and treated at an amplitude of $13 \mu \mathrm{m}$ (d). The circles highlight the agglomerates of CNTs. 
electrical resistivity occurred after ultrasonic treatment. With better dispersion of CNTs, a more robust CNTs network was formed and thus the electrical resistivity was decreased. At relatively high CNTs concentration of 0.8 wt\%, very little difference in dispersion can be seen before and after ultrasonic treatment from Fig. 9(c) and (d). As a result, very limited change of the electrical resistivity occurred, as can be seen from Figs. 4(a) and 5(a).

The TEM micrographs of LPC-0.2CNTs and LPC-0.8CNT samples untreated and treated at an amplitude of 13 $\mu \mathrm{m}$ are shown in Fig. 10. The results were similar to HPC composites, i.e. the dispersion of CNTs was obviously improved at $0.2 \mathrm{wt} \%$ after ultrasonic treatment, while limited effect was observed in $0.8 \mathrm{wt} \%$ samples. This result was consistent with a lower electrical resistivity in $0.2 \mathrm{wt} \%$ samples after ultrasonic treatment, as shown in Figs. 4(b) and 5(b).
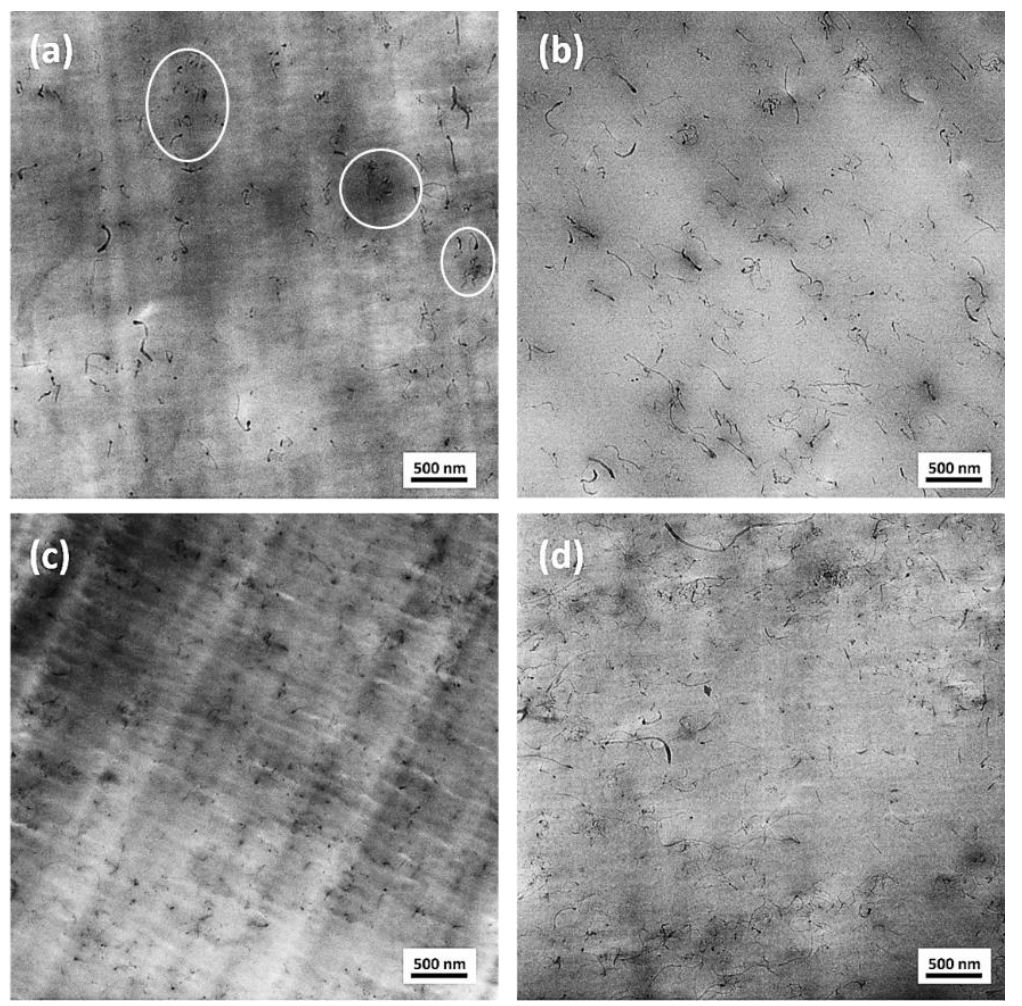

Fig. 9. The TEM micrographs of HPC-0.2CNT untreated (a) and treated at an amplitude of $13 \mu \mathrm{m}$ (b), HPC-0.8CNT untreated (c) and treated at an amplitude of $13 \mu \mathrm{m}(\mathrm{d})$. The circles highlight the agglomerates of CNTs. 

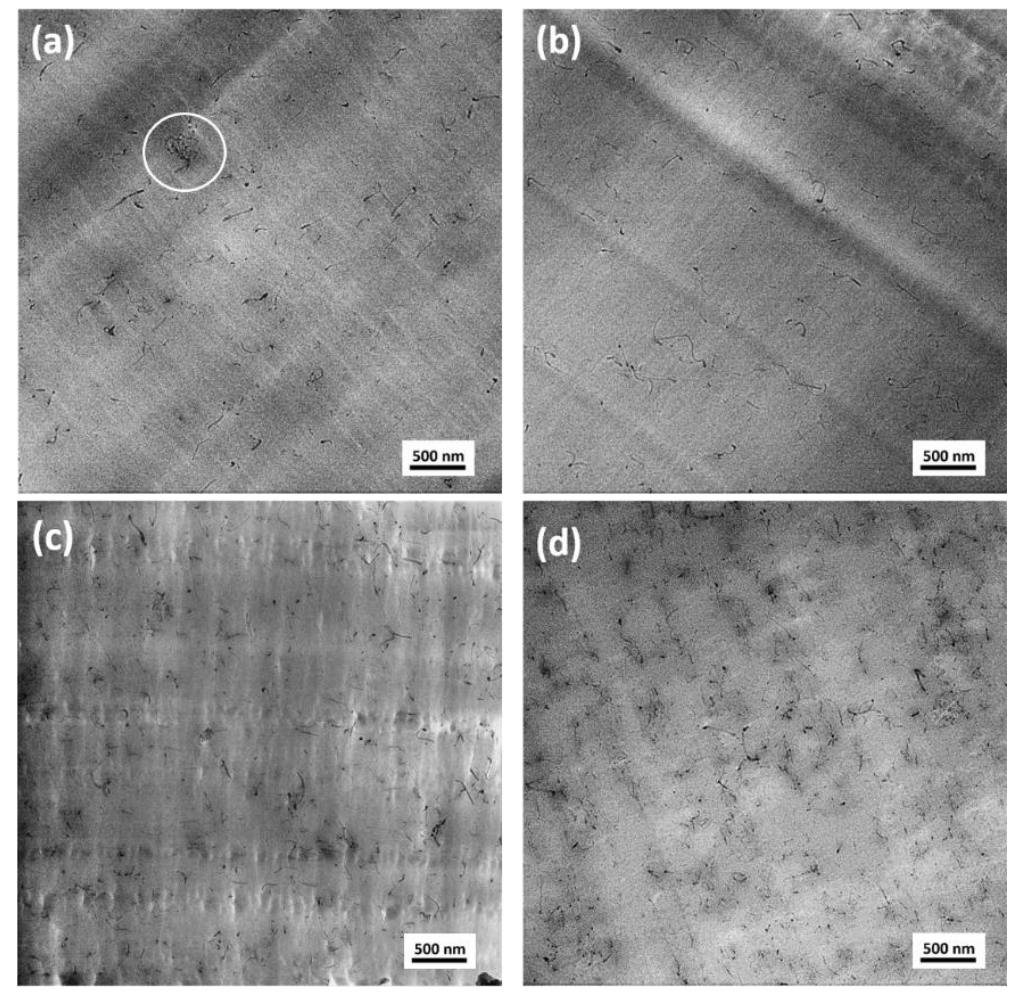

Fig. 10. The TEM micrographs of LPC- $0.2 C N T$ untreated (a) and treated at an amplitude of $13 \mu \mathrm{m}$ (b), LPC- $0.8 C N T$ untreated (c) and treated at an amplitude of $13 \mu \mathrm{m}(\mathrm{d})$. The circle highlights the agglomerates of CNTs.

\subsection{Rheological properties}

The rheological behavior of composites is considered as an indirect but efficient method to determine the dispersion state of nanofillers in polymer composites. Especially, the rheological behavior at low frequency range of small-amplitude oscillatory shear (SAOS) test is highly influenced by the filler-filler and polymer-filler interactions in polymer-nanofillers composites [38,39].

Fig. 11 shows the effect of ultrasound on the storage modulus and Tan $\delta$ of HPC composites as a function of frequency at different CNTs loadings. In Fig. 11(a), typical terminal behavior with the scaling properties of approximately $\mathrm{G}^{\prime} \sim \omega^{2}$ was observed for pure HPC samples. With the increase of CNTs concentration, this terminal behavior started to disappear at $0.2 \mathrm{wt} \%$, which indicated that long-range motion of polymer chains was restrained by the presence of CNTs. Additionally, with the increase of CNTs concentration, the storage modulus consistently increased and the slope of $\mathrm{G}^{\prime}$ at low frequencies decreased. This is a solid evidence of the formation of CNTs network and the increasing interaction between polymer and CNTs. At high frequencies, CNTs showed limited 

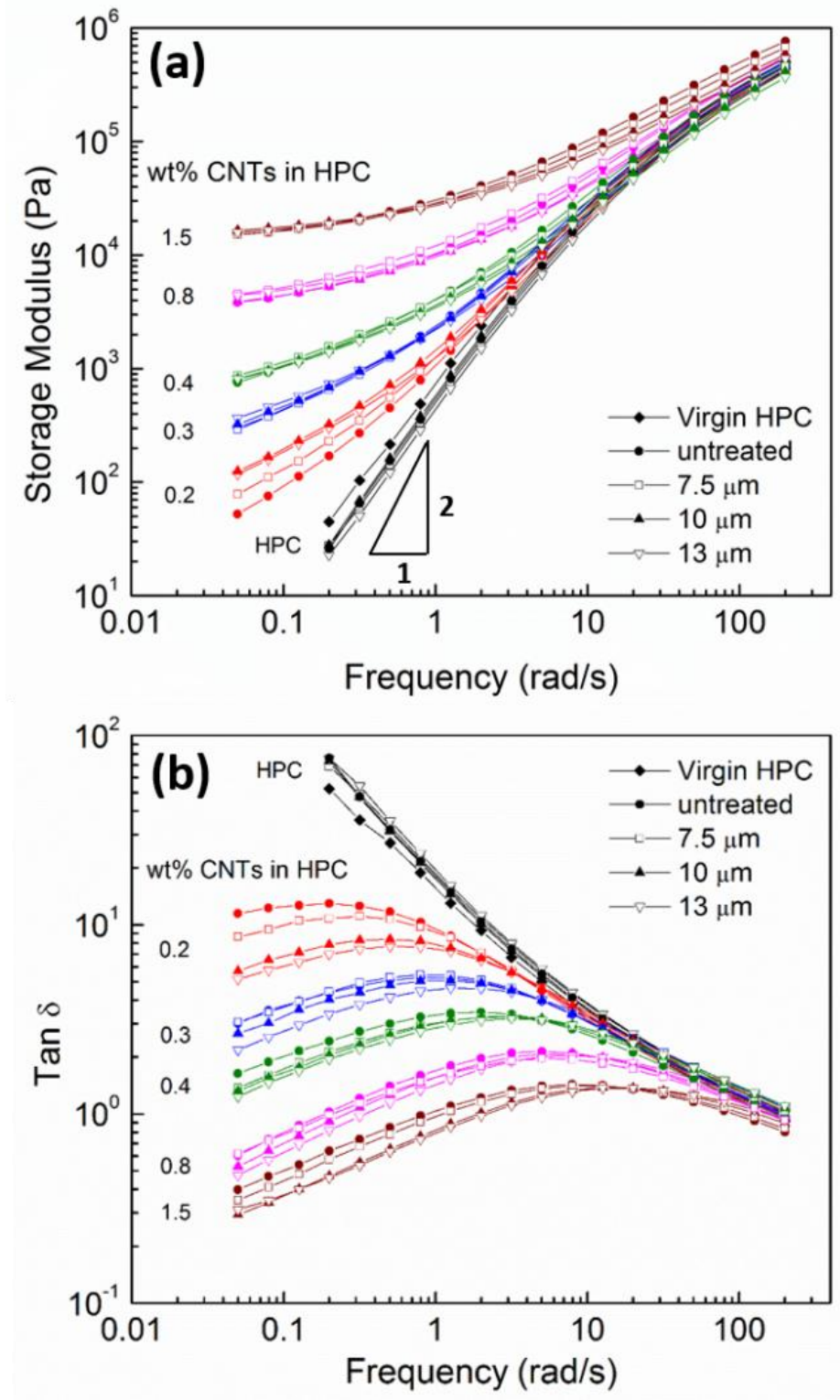

Fig. 11. Storage modulus (a) and $\operatorname{Tan} \delta(b)$ of HPC composites.

effects on the rheological behaviors, which suggested that CNTs did not significantly influence the short-range dynamic motion of polymer chains. From Fig. 11(b), significant decrease of Tan $\delta$ with increasing CNTs concentration was observed at low frequencies, which was also an indication of the effect of CNTs on the relaxation behavior of polymer chains.

The influence of ultrasonic treatment on the rheological properties can also be seen from Fig. 11. In Fig 11(a), the storage modulus of untreated HPC was lower than that of virgin HPC, indicating that certain degradation of polymer happened during extrusion. With the increase of ultrasonic amplitude, further decrease of G' was observed, which 
was caused by the polymer chain scission during ultrasonic treatment. As can be seen from Fig. 11(a), for 0.2 wt $\%$ samples, the storage modulus at low frequencies continuously increased with the increase of ultrasonic amplitude, indicating the improvement of filler dispersion and formation of more robust CNTs networks in the composites after application of ultrasound. The effect of ultrasound on G' at low frequencies became less obvious for $0.3 \mathrm{wt} \%$ and higher CNTs concentrations samples. It should be noted that when ultrasound was applied during processing, two effects occurred. One is the chain scission of the polymer chains and the other is the breakup of the CNTs agglomerates. Accordingly, the storage modulus was a result of the competition of these two effects. So it is reasonable to say that for samples with CNTs concentration higher than $0.3 \mathrm{wt} \%$, the effect of ultrasound on improving the dispersion of CNTs was possibly concealed by the degradation of polymer chains. It can be seen from Fig 11(b) that Tan $\delta$ at low frequencies decreased with the increase of ultrasonic amplitude for samples at same CNTs concentration. This was because the storage modulus increased more than the loss modulus after ultrasonic treatment. The lower Tan $\delta$ for ultrasonically treated samples indicated improved interaction between polymer and CNTs and more robust CNTs network formed with the increase of the ultrasonic amplitude.

For pure LPC, there was no obvious decrease of the storage modulus (as can be seen in Fig. 12(a)). The storage modulus difference between HPC and LPC after ultrasonic treatment was probably due to lower processing temperature and lower shear stress. This correlated with lower torque in Fig. 3(b) during the extrusion of LPC composites.

LPC composites showed similar behavior of the storage modulus and Tan $\delta$ as HPC composites. The storage modulus G' at low frequencies increased and the slope of G' decreased with increasing concentration of CNTs. At lower concentration of CNTs, e.g. $0.2 \mathrm{wt} \%, 0.3 \mathrm{wt} \%, 0.4 \mathrm{wt} \%$, the effect of ultrasound can be obviously observed from the increase of the storage modulus with increasing ultrasonic amplitude. Especially, for the LPC/CNTs composites at $0.2 \mathrm{wt} \% \mathrm{CNTs}$, the storage modulus at the frequency of $0.05 \mathrm{rad} / \mathrm{s}$ showed an increase of almost one order of magnitude at an ultrasonic amplitude of $7.5 \mu \mathrm{m}$. When comparing this result with that of HPC-0.2CNT sample, ultrasound showed larger effect on LPC composites than HPC composites at $7.5 \mu \mathrm{m}$. From Tan $\delta$ at low frequencies in Fig 12(b), it was confirmed that ultrasound had larger effect on LPC-0.2CNT on improving dispersion of CNTs and polymer-filler interaction. 

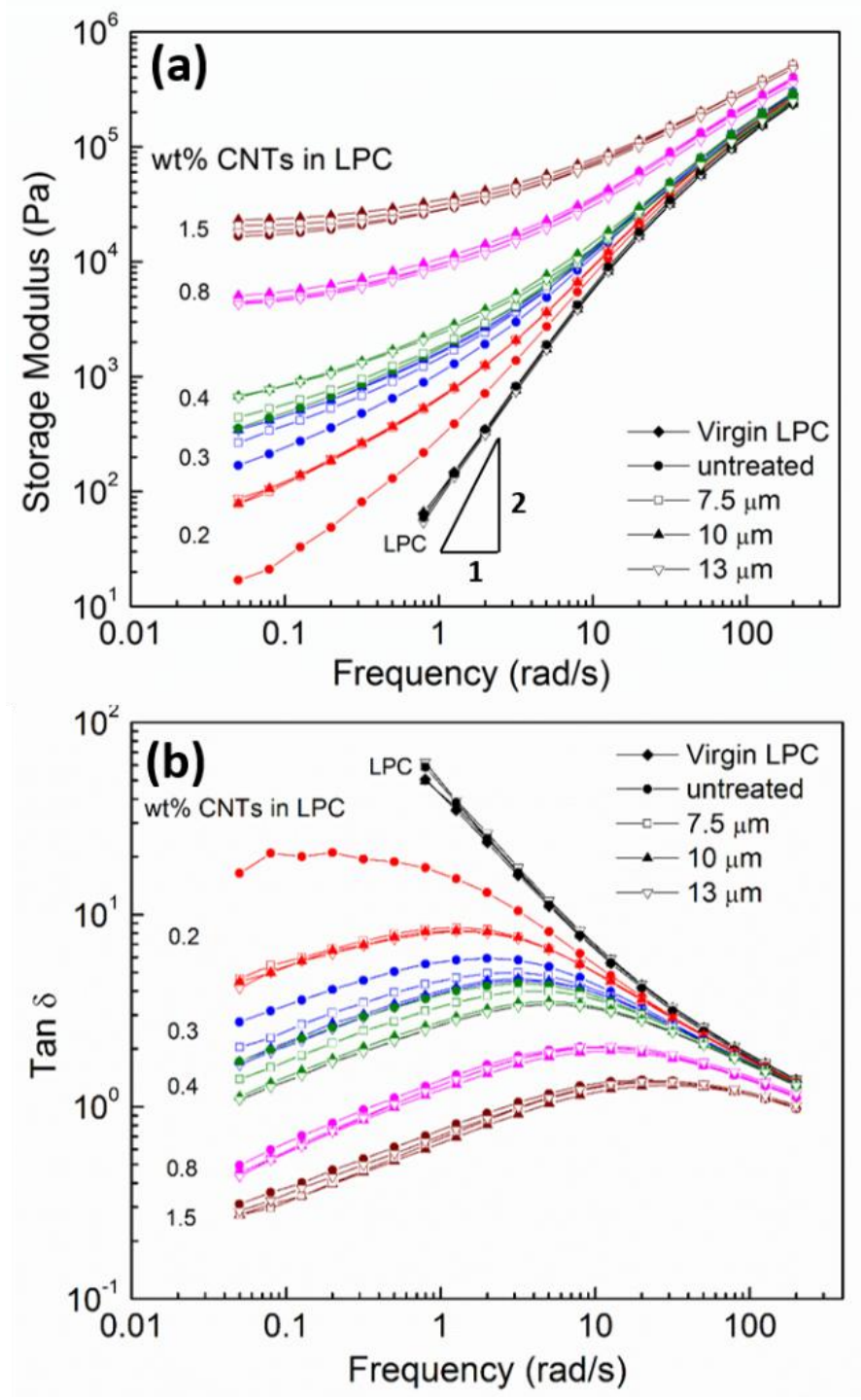

Fig. 12. Storage modulus (a) and Tan $\delta$ (b) of LPC composites.

As mentioned above, the effect of ultrasonic treatment on the dispersion of CNTs might be concealed by the degradation of polymer matrix, so modified Cole-Cole plot (i.e. log G” vs log G') was used to reveal the structure variation in the composites while "hiding" the effect of molecular weight. The Cole-Cole plot of polymers varying only in molecular weight can be reduced to a single master curve [40,41]. Therefore, it has been proved to be an efficient method to reveal the change in structure of polymer or polymer composites that are caused by molecular weight distribution [42], molecular structure [43,44], and polymer-filler interactions [38,45].

From the Cole-Cole plots in Fig. 13, the data of pure HPC and LPC samples were reduced to a single curve, showing that there was no microstructure difference between untreated and treated PC resins. For the PC/CNTs composites, at a given loss modulus G', the storage modulus G' increased significantly with increase of CNTs 
concentration, indicating obvious changes of the microstructure of the composites. This was attributed to higher PCCNTs interaction, making the composites at high CNTs concentration more elastic. In addition, for PC/CNTs samples at same CNTs concentrations, the Cole-Cole plot shifted towards higher storage modulus with increasing ultrasonic amplitude. This was a solid evidence that the ultrasonic treatment improved the dispersion of CNTs and thus increased the PC-CNTs interactions. Similar to results of the storage modulus and Tan $\delta$ shown in Figs. 11 and 12, ultrasound showed more pronounced effect at low CNTs concentrations and limited effect at high CNTs concentration.
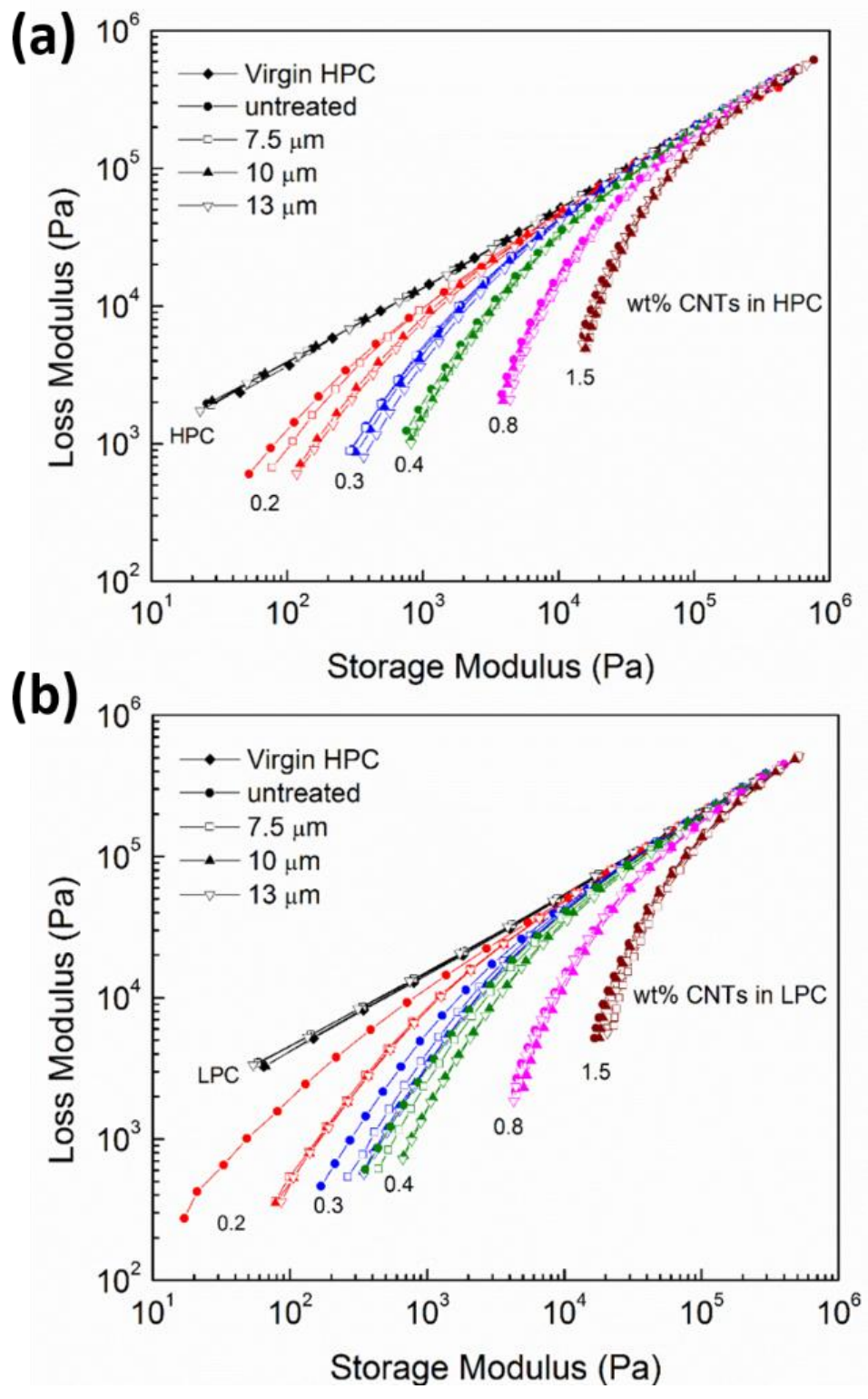

Fig. 13. Cole-Cole plot of HPC (a) and LPC (b) composites. 
As discussed above, with the addition of CNTs, the storage modulus of the composites at low frequencies increased dramatically. When a critical value of concentration was achieved, the storage modulus showed a significant increase. This corresponded to the rheological percolation threshold showing the onset of solid-like behavior. Above the percolation threshold, a power-law relationship between the storage modulus and CNTs volume concentration could be observed [46]:

$$
G^{\prime}(\varphi)=k\left(\varphi-\varphi_{0}\right)^{v}
$$

where $\varphi$ is the volume concentration of CNTs, $\varphi_{0}$ is the rheological percolation threshold, $v$ is the exponent, G' is the storage modulus at the frequency of $0.05 \mathrm{rad} / \mathrm{s}$ in this experiment. $v=2$ was used in the fitting for the same reason as mentioned above.

Composites with CNTs concentration above the percolation threshold, which is equal or above 0.118 vol\%, were chosen to fit the storage modulus to Eq. (3). The storage modulus of both HPC and LPC composites as a function of the volume concentration were fitted to Eq. (3) and the results are shown in Fig. 14. The single data point at the left bottom corner shows the storage modulus of virgin PC. A large increase of G' can be seen when percolation happens, which indicated that a sudden structure change in the material happened and CNTs formed a percolated network impeding the motion of polymer chains [46]. Samples treated at an ultrasonic amplitude of $13 \mu \mathrm{m}$ were used as an example to show a power law fit of Eq. (3) to the data in the inset figure. The fitting parameters are summarized in Table 3. The rheological percolation thresholds in Tables 3 are plotted in Fig. 15.

As shown in Fig. 15, the rheological percolation threshold continuously decreased with the increase of ultrasonic amplitude. The $\varphi_{0}$ of HPC composites decreased from 0.0619 vol $\%$ for untreated samples to 0.0439 vol\% for treated samples at an amplitude of $13 \mu \mathrm{m}$, and $\varphi_{0}$ of LPC composites decreased from 0.102 vol\% for untreated samples to $0.0550 \mathrm{vol} \%$ for treated samples at an amplitude of $13 \mu \mathrm{m}$. Ultrasound showed larger effect on improving the dispersion of CNTs in LPC composite, which was indicated by $45 \%$ decrease of the rheological percolation threshold. 

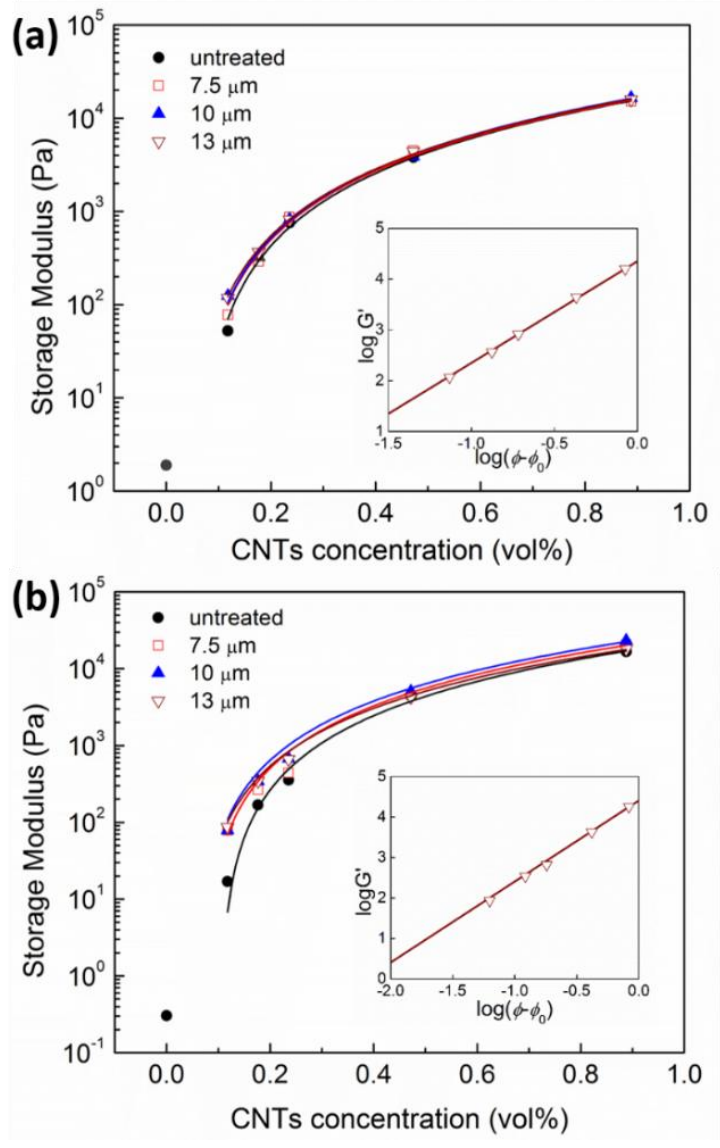

Fig. 14. Storage modulus of HPC (a) and LPC (b) composites as a function of the volume concentration of CNTs at different ultrasonic amplitudes. Inset figures show a power law fit of Eq. (3) to the data of PC composites treated at an amplitude of $13 \mu \mathrm{m}$. The single data point at the left bottom corner is the storage modulus of virgin PC.

Table 3. Fitting parameters of Eq. (3) for HPC and LPC composites.

\begin{tabular}{|c|c|c|c|c|c|}
\hline & & $0 \mu \mathrm{m}$ & $7.5 \mu \mathrm{m}$ & $10 \mu \mathrm{m}$ & $13 \mu \mathrm{m}$ \\
\hline \multirow{3}{*}{ HPC } & $\mathrm{k}(\mathrm{Pa})$ & 22482 & 22150 & 23215 & 22493 \\
\hline & $\varphi_{0}(\operatorname{vol} \%)$ & 0.0619 & 0.0498 & 0.0502 & 0.0439 \\
\hline & $\mathrm{R}^{2}$ & 0.99898 & 0.99178 & 0.99837 & 0.99836 \\
\hline \multirow{3}{*}{ LPC } & $\mathrm{k}(\mathrm{Pa})$ & 27531 & 30091 & 32744 & 25672 \\
\hline & $\varphi_{0}(\operatorname{vol} \%)$ & 0.102 & 0.0701 & 0.0612 & 0.0550 \\
\hline & $\mathrm{R}^{2}$ & 0.99036 & 0.99589 & 0.99409 & 0.99874 \\
\hline
\end{tabular}




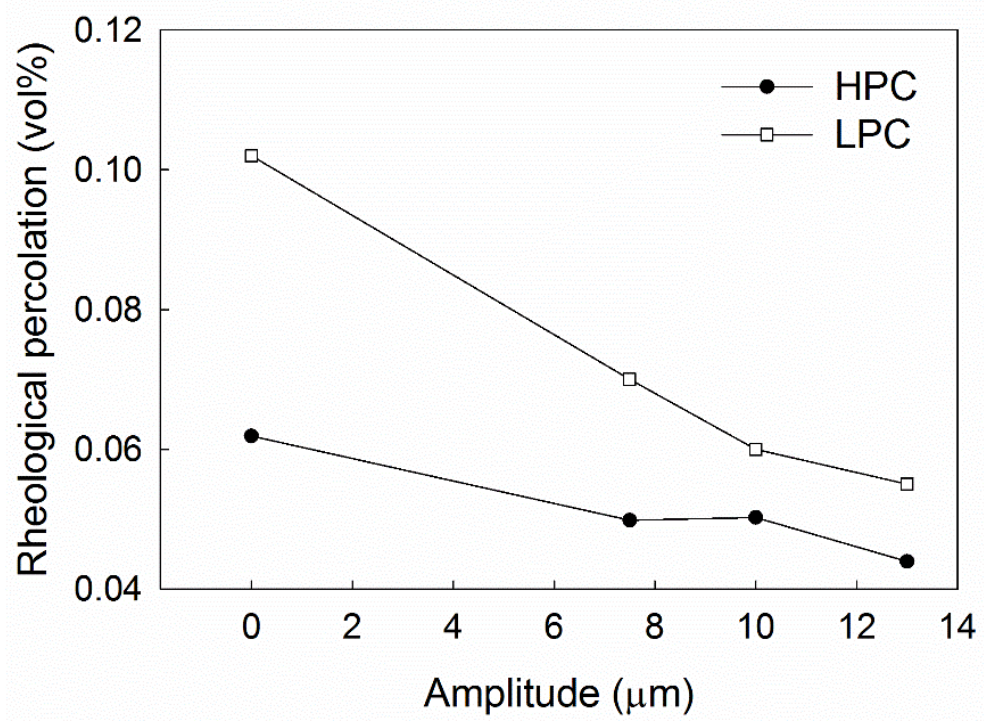

Fig. 15. Rheological percolation threshold as a function of ultrasonic amplitude of HPC and LPC composites.

When comparing the rheological and electrical percolation threshold from Figs. 7 and 15, it is worthwhile to note that the rheological percolation threshold is lower than electrical threshold for the samples at the same conditions. For example, one can see that for untreated HPC/CNTs composites, the rheological percolation is 0.0619 vol\%, which is only half of the electrical percolation $(0.135 \mathrm{vol} \%)$.This can be explained by the different filler-filler distances required for rheological and electrical percolation [46]. In the PC/CNTs composites, electron hopping mechanism is applied to the electrical conductivity, the required filler-filler distance is less than $5 \mathrm{~nm}$ for the composites to be conductive. On the other hand, as long as the filler-filler distance is comparable to the diameter of random coils of PC chains, the CNTs network can effectively restrain polymer motion. The average radius of gyration of PC chains can be calculated as:

$$
R_{w}=\beta M_{w}^{\alpha}
$$

where $R_{w}$ is the mean-squared radius of gyration with a unit of $\AA, \mathrm{M}_{\mathrm{w}}$ is the weight average molecular weight of PC, $\beta=0.457, \alpha=0.5$ according to [47]. Based on Eq. (4), the average diameter of the random coils $2 R_{w}$ for HPC and LPC are $16.1 \mathrm{~nm}$ and $12.1 \mathrm{~nm}$, respectively. Therefore, the required filler-filler distance for rheologcial percolation is much larger than that for the electrical percolation. As a result, more CNTs are required to reach the electrical conductivity threshold in the composites. Also, it should be noted that CNTs could be metallic or non-metallic due to the difference in CNTs chirality determining the electrical behavior of CNTs [48]. Therefore, in the PC/CNTs composites non-metallic CNTs do not contribute to the electrically conductive network, though they could restrict 
the motion of polymer chains. Furthermore, it can be seen from Fig. 15 that the rheological percolation threshold of HPC is lower than that of LPC. This can be explained by the larger filler-filler distance required for HPC composites to restrict the polymer chain motion than LPC composites.

Overall, ultrasonic treatment showed larger effect in LPC composites than HPC composites in the electrical and rheological properties, which was mainly due to the better CNTs dispersion in LPC composites. One reason is that ultrasonic power consumption during extrusion of LPC composites is higher than that of HPC composites, indicating more energy was transmitted into polymer melt to break the agglomerates of CNTs. Also due to the low viscosity of LPC during ultrasonic treatment, the cavitating bubble in the polymer melt can be easily expanded and contracted by ultrasound to break the agglomerates. Another reason is that the shear stress during the extrusion of LPC composites is lower than that of HPC composites. Therefore, the scission of nanotubes happened less in LPC composites and the aspect ratio of nanotubes was generally higher than HPC composites [27]. As a result, electrical and rheological percolations of LPC composites were more significantly decreased.

\subsection{Mechanical properties}

The Young's modulus, yield stress, elongation at break and tensile strength of HPC composites untreated and treated at an amplitude of $13 \mu \mathrm{m}$ as a function of CNTs concentration are shown in Fig. 16. The Young's modulus and yield stress generally increased with the increase of CNTs concentration. The significant improvement of Young's modulus at low CNTs concentration was possibly due to the formation of the CNTs network and the immobilization of HPC molecular chains on the surface of nanotubes. Ding et al. [49] found that molecular chains of PC can form a sheathing layer on nanotubes, which can efficiently enhance the interaction and load transfer between PC and CNTs. As a result, the Young's modulus was improved after incorporation of CNTs. A drop of yield stress was observed in HPC-0.2CNT samples, which was probably due to the incorporation of CNTs causing defects influencing the yield behavior of the polymer. The elongation at break decreased with the increase of CNTs concentration. Higher content of CNTs might induce more defects in the samples leading to lower elongation. The tensile strength showed similar decreasing trend as the elongation at break with CNTs concentration. It should be noted that the samples generally broke up at the strain hardening region after necking. As a result, the lower the

elongation at break, the lower the tensile strength. Thus the tensile strength showed similar trend as the elongation at break. The ultrasonically treated HPC/CNTs composites at an amplitude of $13 \mu \mathrm{m}$ generally showed a higher 
Young's modulus and elongation at break than untreated samples. The increase of these properties after ultrasonic treatment was mainly caused by the improvement of CNTs dispersion and thus more robust CNTs network and less defects.

The Young's modulus, yield stress, elongation at break and tensile strength of LPC composites untreated and treated at an amplitude of $13 \mu \mathrm{m}$ as a function of CNTs concentration are shown in Fig. 17. Similar to the results of HPC composites, the Young's modulus and yield stress of LPC composites generally increased with CNTs concentration, while the elongation at break continuously decreased. This observation for LPC composites can be explained in the same way as that for HPC composites. Taking the error bar into consideration, there is almost no change in the tensile strength of LPC composites with CNTs concentration. This is because the samples of LPC composites were failed in the necking region. As a result, there is no large difference between samples at different concentrations. The effect of ultrasonic treatment can be clearly observed from the higher Young's modulus in treated samples.
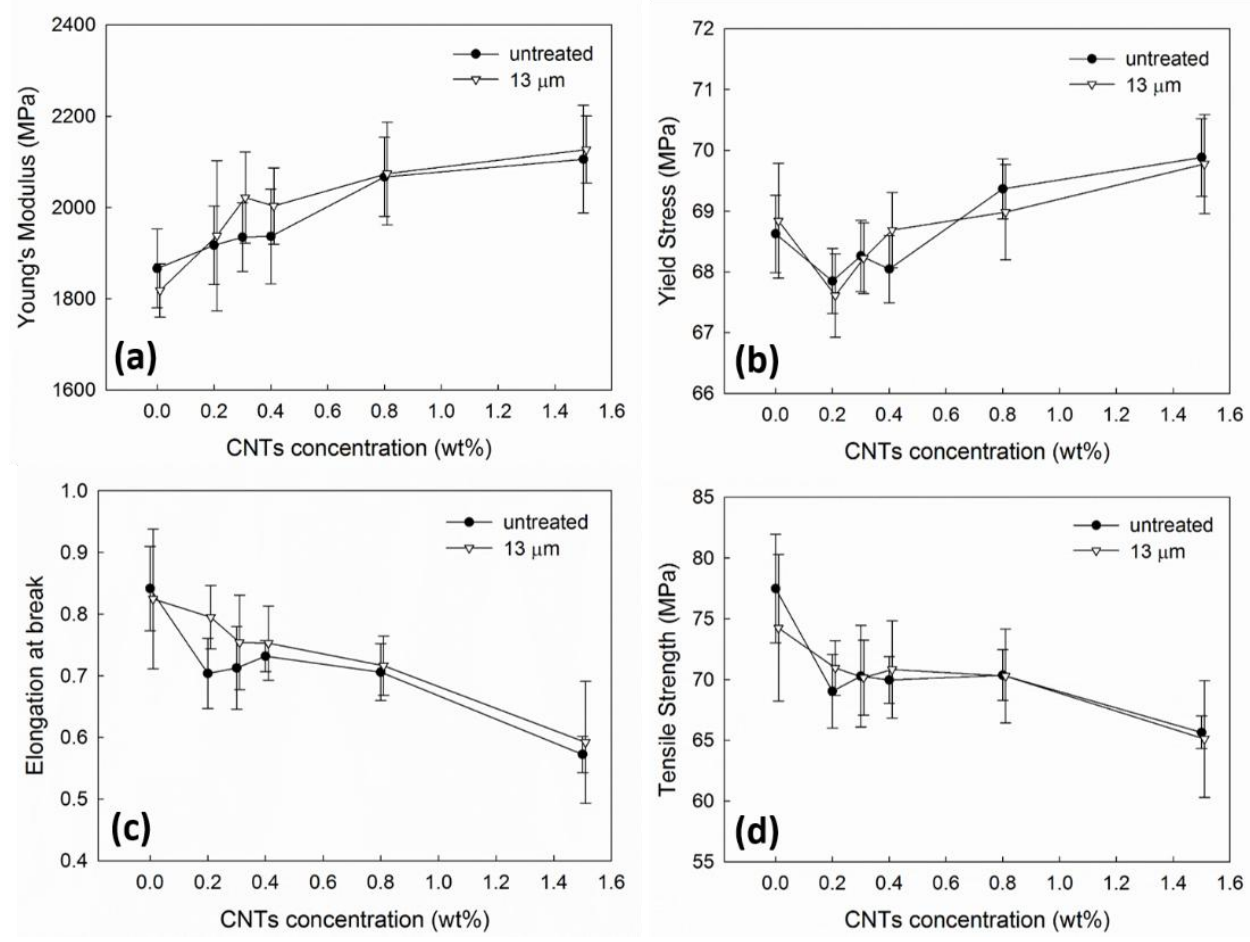

Fig. 16. Young's modulus (a), yield stress (b), elongation at break (c) and tensile strength (d) of HPC composites as a function of CNTs concentration. The data of samples treated at $13 \mu \mathrm{m}$ were slightly shifted along abscissa to more clearly show the error bars. 

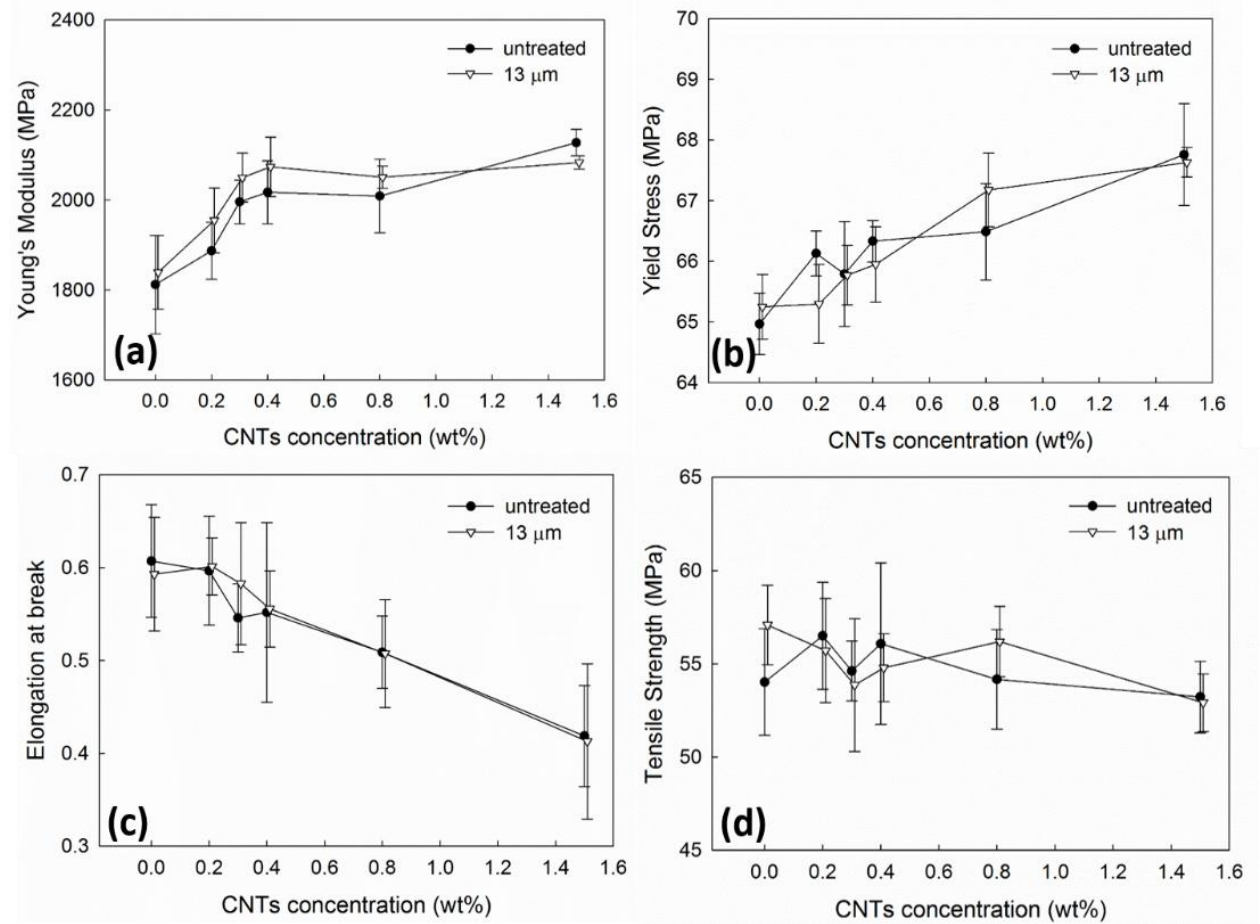

Fig. 17. Young's modulus (a), yield stress (b), elongation at break (c) and tensile strength (d) of LPC composites as a function of CNTs concentration. The data of samples treated at $13 \mu \mathrm{m}$ were slightly shifted along abscissa to more clearly show the error bars.

When comparing HPC and LPC samples at the same CNTs concentrations and ultrasonic amplitudes, it can be seen that HPC samples showed higher yield stress, elongation at break and tensile strength. According to work by Gardner and Martin [50] and Pitman et al. [51], the stress required for craze initiation decreases as the molecular weight decreases. Therefore, LPC samples showed a lower yield stress. It was also reported by Gardner and Martin [50] that the elongation at break decreased dramatically as the molecular weight is reduced. Accordingly, the LPC samples showed lower elongation and tensile strength.

\subsection{Mechanism of ultrasonic effect on electrical and rheological percolations}

From the above discussion, it can be seen that ultrasound showed tremendous effect on the rheological and electrical properties of the PC composites at low CNTs concentration $(0.2 \mathrm{wt} \%, 0.3 \mathrm{wt} \%$ and $0.4 \mathrm{wt} \%)$ but limited effect on higher CNTs concentration composites. This was proven by the large increase of the storage modulus at lower frequency, the significant decrease of electrical surface and volume resistivity and the improvement of mechanical properties. Also, the electrical and rheological percolation threshold largely decreased with the increase of the ultrasonic amplitude. All these results indicated that a larger effect of ultrasound can be easily observed at low 
CNTs concentration. Based on these findings, the following mechanism for this effect was proposed. This is schematically shown in Fig. 18. At low filler concentration, CNTs agglomerates and bundles are loosely dispersed in the polymer matrix. In this case, the density of the fillers is not high enough to form a percolated network. However, after ultrasound treatment, the CNTs agglomerates are broken up by the high power ultrasound. With such an improvement of CNTs dispersion and distribution, a percolated network is formed. On the other hand, for the composites with high CNTs concentration, a percolated network is already formed, though most of the fillers are in the state of agglomerates. After ultrasonic treatment, the agglomerates are broken up and the dispersion of fillers are improved, but this improvement is not easy to observe from the rheological or electrical properties because the filler network is already percolated before the ultrasound treatment.
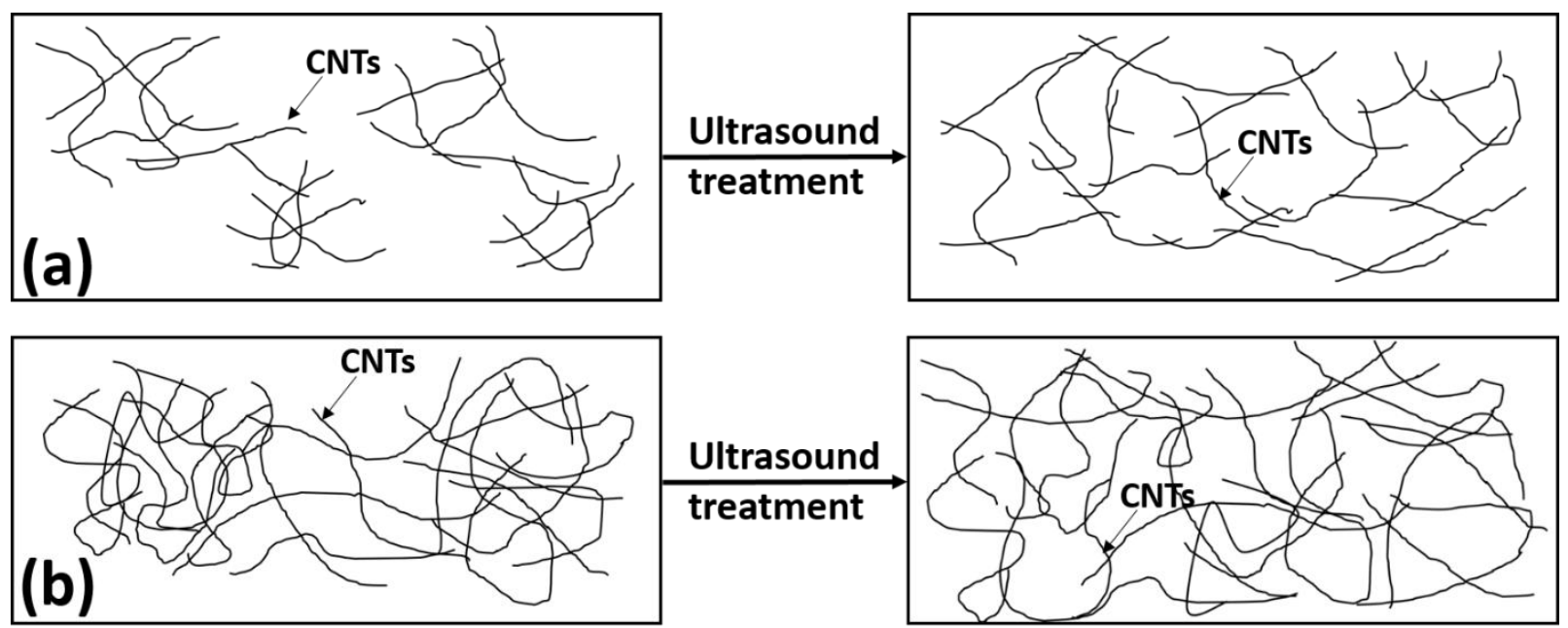

Fig. 18. Schematic of the effect of ultrasonic treatment on low (a) and high (b) CNTs concentration composites.

\section{Conclusions}

Ultrasonically aided twin-extruder was employed to prepare HPC/CNTs and LPC/CNTs composites without and with the imposition of ultrasonic waves. With the increase of ultrasonic amplitude, the power consumption continuously increased and the torque of the extruder decreased. CNTs concentration showed little effect on the power consumption and torque. The surface and volume resistivity of the composites exhibited typical percolation behavior with the increase of CNTs concentration and the electrical percolation threshold decreased tremendously with the increase of ultrasonic amplitude. This was found to be caused by the improved CNTs dispersion and distribution in the composites as shown by the optical microscopy and TEM studies. The storage modulus at low 
frequency increased with the increase of ultrasonic amplitude and the rheological percolation threshold for both HPC and LPC composites significantly decreased with ultrasonic treatment. When comparing the rheological and electrical percolation threshold, it was found that the rheological percolation threshold was lower than the electrical threshold, which can be attributed to the shorter filler-filler distance required to restrain the motion of polymer chains and that the nonmetallic part of the CNTs could not contribute to the electrical conductive path in the composites. Obvious improvement in mechanical properties after ultrasound treatment at an amplitude of $13 \mu \mathrm{m}$ was observed. Finally, a mechanism was proposed to explain the decrease of the rheological and electrical percolation. At low CNTs concentration, the originally not percolated CNTs agglomerates were broken up after ultrasonic treatment and formed a percolated CNTs network. On the other hand, this effect was not easy to be observed for high CNTs concentration composites which already had a percolated CNTs network before ultrasonic treatment.

\section{Acknowledgements}

The authors wish to acknowledge the NSF Division of Engineering for financial support under grant CMMI1131342 and SABIC plastics for providing PC resins. The authors also thank Dr. Xiong Gong for the help in the surface resistivity test, Dr. Wei Sen Wong from Malvern Instruments for the help with GPC measurements, Mr. Ming Xiao from The University of Akron and Mr. Yongsheng Zhao from Sichuan University for their help with TEM.

\section{References}

1. Iijima S. Nature 1991;354(6348):56-58.

2. Mai F, Pan D, Gao X, Yao M, Deng H, Wang K, Chen F, and Fu Q. Polymer International 2011;60(11):1646-1654.

3. Gao X, Zhang S, Mai F, Lin L, Deng Y, Deng H, and Fu Q. Journal of Materials Chemistry 2011;21(17):6401-6408.

4. Z Zhong J, Isayev AI, and Huang K. Polymer 2014;55(7):1745-1755.

5. Müller MT, Pötschke P, and Voit B. Polymer 2015;66:210-221.

6. Shui J, Wang M, Du F, and Dai L. Science Advances 2015;1(1).

7. Wong EW, Sheehan PE, and Lieber CM. Science 1997;277(5334):1971-1975.

8. Yu M-F, Lourie O, Dyer MJ, Moloni K, Kelly TF, and Ruoff RS. Science 2000;287(5453):637-640. 
9. $\quad$ Liu K, Sun Y, Lin X, Zhou R, Wang J, Fan S, and Jiang K. ACS Nano 2010;4(10):5827-5834.

10. Berber S, Kwon Y-K, and Tománek D. Physical Review Letters 2000;84(20):4613-4616.

11. Bauhofer W and Kovacs JZ. Composites Science and Technology 2009;69(10):1486-1498.

12. Moniruzzaman M and Winey KI. Macromolecules 2006;39(16):5194-5205.

13. Lee H-J, Oh S-J, Choi J-Y, Kim JW, Han J, Tan L-S, and Baek J-B. Chemistry of Materials 2005;17(20):5057-5064.

14. Park SJ, Cho MS, Lim ST, Choi HJ, and Jhon MS. Macromolecular Rapid Communications 2003;24(18):1070-1073.

15. Liu K, Chen L, Chen Y, Wu J, Zhang W, Chen F, and Fu Q. Journal of Materials Chemistry 2011;21(24):8612-8617.

16. Chen H, Huang C, Yu W, and Zhou C. Polymer 2013;54(6):1603-1611.

17. Kaida S, Matsui J, Sagae T, Hoshikawa Y, Kyotani T, and Miyashita T. Carbon 2013;59(0):503-511.

18. Tung VC, Chen L-M, Allen MJ, Wassei JK, Nelson K, Kaner RB, and Yang Y. Nano Letters 2009;9(5):1949-1955

19. Wang H, Hsieh B, Jiménez-Osés G, Liu P, Tassone CJ, Diao Y, Lei T, Houk KN, and Bao Z. Small 2015;11(1):126-133.

20. Choi J and Isayev AI. Rubber Chemistry and Technology 2012;85(1):14-37.

21. Isayev AI, Kumar R, and Lewis TM. Polymer 2009;50(1):250-260.

22. Swain SK and Isayev AI. Polymer 2007;48(1):281-289.

23. Kumar R and Isayev AI. Polymer 2010;51(15):3503-3511.

24. Niknezhad S and Isayev AI. Journal of Applied Polymer Science 2013;129(1):263-275.

25. Zhong J and Isayev AI. Journal of Applied Polymer Science 2014;132(5):DOI: 10.1002/app.41397.

26. Villmow T, Kretzschmar B, and Pötschke P. Composites Science and Technology 2010;70(14):2045-2055.

27. Socher R, Krause B, Müller MT, Boldt R, and Pötschke P. Polymer 2012;53(2):495-504.

28. Castillo FY, Socher R, Krause B, Headrick R, Grady BP, Prada-Silvy R, and Pötschke P. Polymer 2011;52(17):3835-3845.

29. Krause B, Boldt R, Häußler L, and Pötschke P. Composites Science and Technology 2015;114:119-125. 
30. Guo J, Liu Y, Prada-Silvy R, Tan Y, Azad S, Krause B, Pötschke P, and Grady BP. Journal of Polymer Science Part B: Polymer Physics 2014;52(1):73-83.

31. Long TS and Sokol RJ. Polymer Engineering \& Science 1974;14(12):817-822.

32. Huang K and Isayev AI. Polymer 2015;70:290-306.

33. Yashin VV and Isayev AI. Rubber Chemistry and Technology 1999;72(4):741-757.

34. Coleman JN, Curran S, Dalton AB, Davey AP, McCarthy B, Blau W, and Barklie RC. Physical Review B 1998;58(12):R7492-R7495.

35. Isichenko MB. Reviews of Modern Physics 1992;64(4):961-1043.

36. Alig I, Pötschke P, Lellinger D, Skipa T, Pegel S, Kasaliwal GR, and Villmow T. Polymer 2012;53(1):428.

37. Pötschke P, Bhattacharyya AR, and Janke A. European Polymer Journal 2004;40(1):137-148.

38. Pötschke P, Fornes TD, and Paul DR. Polymer 2002;43(11):3247-3255.

39. Pötschke P, Abdel-Goad M, Alig I, Dudkin S, and Lellinger D. Polymer 2004;45(26):8863-8870.

40. Han CD and Jhon MS. Journal of Applied Polymer Science 1986;32(3):3809-3840.

41. Harrell ER and Nakajima N. Journal of Applied Polymer Science 1984;29(3):995-1010.

42. Han CD. Journal of Applied Polymer Science 1988;35(1):167-213.

43. Tian J, Yu W, and Zhou C. Polymer 2006;47(23):7962-7969.

44. Isayev AI, Liang T, and Lewis TM. Rubber Chemistry and Technology 2014;87(1):86-102.

45. Golba JC, Kunzelman J, and Spikowski JM. SPE ANTEC paper 2012.

46. Du F, Scogna RC, Zhou W, Brand S, Fischer JE, and Winey KI. Macromolecules 2004;37(24):9048-9055.

47. Ballard DGH, Burgess AN, Cheshire P, Janke EW, Nevin A, and Schelten J. Polymer 1981;22(10):13531354.

48. $\quad$ Ebbesen TW, Lezec HJ, Hiura H, Bennett JW, Ghaemi HF, and Thio T. Nature 1996;382(6586):54-56.

49. Ding W, Eitan A, Fisher FT, Chen X, Dikin DA, Andrews R, Brinson LC, Schadler LS, and Ruoff RS. Nano Letters 2003;3(11):1593-1597.

50. Gardner RJ and Martin JR. Journal of Applied Polymer Science 1979;24(5):1269-1280.

51. Pitman GL, Ward IM, and Duckett RA. Journal of Materials Science 1978;13(10):2092-2104. 